\title{
Viscoelastic property tuning for reducing noise radiated by switched-reluctance machines
}

\author{
Pierre Millithaler $^{\mathrm{a}, \mathrm{b}}$, Jean-Baptiste Dupont ${ }^{\mathrm{a}}$, Morvan Ouisse ${ }^{\mathrm{b}}$, Émeline Sadoulet-Reboul $^{\mathrm{b}}$, Noureddine \\ Bouhaddi $^{\mathrm{b}}$ \\ ${ }^{a}$ Vibratec, 28 chemin du petit bois, BP36, F-69131 Écully Cedex, FRANCE \\ ${ }^{b}$ Univ. Bourgogne Franche-Comté, FEMTO-ST Institute, CNRS/UFC/ENSMM/UTBM, Department of Applied Mechanics, \\ 25000 Besanon, FRANCE
}

\begin{abstract}
Switched-reluctance motors (SRM) present major acoustic drawbacks that hinder their use for electric vehicles in spite of widely-acknowledged robustness and low manufacturing costs. Unlike other types of electric machines, a SRM stator is completely encapsulated/potted with a viscoelastic resin. By taking advantage of the high damping capacity that a viscoelastic material has in certain temperature and frequency ranges, this article proposes a tuning methodology for reducing the noise emitted by a SRM in operation. After introducing the aspects the tuning process will focus on, the article details a concrete application consisting in computing representative electromagnetic excitations and then the structural response of the stator including equivalent radiated power levels. An optimised viscoelastic material is determined, with which the peak radiated levels are reduced up to $10 \mathrm{~dB}$ in comparison to the initial state. This methodology is implementable for concrete industrial applications as it only relies on common commercial finite-element solvers.
\end{abstract}

Keywords: viscoelastic behaviour, switched-reluctance machine, encapsulation resin, coupled electromagnetic-structural response simulation, acoustic emission

\section{Introduction}

For both energy and environmental reasons, the automotive industry opts more and more for hybrid or 100\%-electric powertrain technologies. Amongst the different types of electric machines (direct-current, synchronous, asynchronous, ...), the so-called Switched-Reluctance Motor (SRM) is distinguished by a very simple rotor architecture without any windings or permanent magnet. Resulting in low manufacturing costs, this aspect also guarantees robustness to the structure, thus making it attractive for industrial production. However, SRMs have been known for important torque ripple and high noise generation [1] that explain the fact they are currently rather undesirable in car powertrains.

Similarly to an alternate-current (AC) electric machine, the stator of an SRM includes windings in order to generate electromagnetic flux and thus torque when fed with electric currents. Then, the windings are protected by an insulating resin (usually a polymer) in order to prevent short-circuits from taking place between the copper wires. But whereas an AC machine's stator often has numerous, thin teeth, an SRM is composed of fewer and wider teeth and slots (see Figure 1). Therefore, this difference requires adapted

Email addresses: pierre.millithaler@gmail.com (Pierre Millithaler), jean-baptiste.dupont@vibratec.fr (Jean-Baptiste Dupont), morvan.ouisse@femto-st.fr (Morvan Ouisse), emeline.sadoulet-reboul@univ-fcomte.fr (Émeline Sadoulet-Reboul), noureddine.bouhaddi@univ-fcomte.fr (Noureddine Bouhaddi) 
resin settings for each motor type; while in the former case, windings are impregnated, the latter stator is entirely potted (or encapsulated) with resin [2].

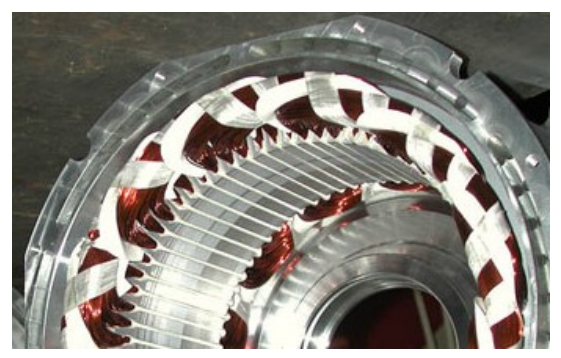

(a): distributed AC motor windings

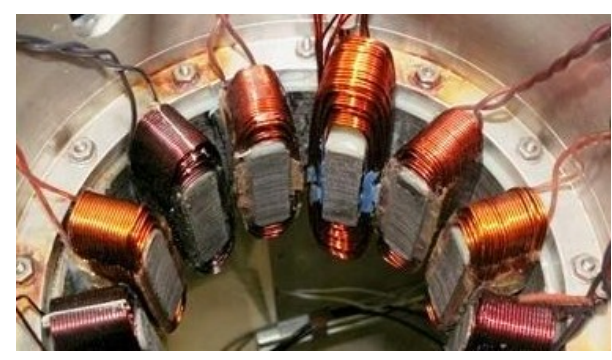

(b): on-tooth SRM windings

Figure 1: AC vs. SRM stator architectures [3, 4]

Concerning the design of potting resins, usual constraints are electric and thermal insulating specifications related to the conditions the electric machine has to sustain while operating [2]. Including new specifications for acoustic performances therefore represents new challenges, but also new opportunities. The first challenge to deal with is the increased complexity that viscoelastic materials represent in comparison to standard elastic properties when performing simulations involving polymers. A brief review of some existing modelling methods in such cases is made in the following paragraph.

Plouin and Balmès [5] have developed a modelling technique for sandwich shell structures including frequency- and temperature-dependent viscoelastic properties. Their application to predict the modal behaviours of flat and curved sandwich composites was successful and coupled to an efficient reduction approach to mitigate the important computation costs of their simulations. However, the use of twodimensional elements in the approach compromises its applicability to general three-dimensional structures.

Slater et al. [6] as well as Vasques et al. [7] reviewed, developed and compared a series of mathematical methods and algorithms designed for modelling viscoelastic effects in finite-element structures, including the approaches detailed in [8]. The influence of stator temperature on an electric machine's NVH behaviour has been observed experimentally by Tan-Kim et al. [9]. The ability to take into account temperature- and/or frequency-dependency in commercial finite-element software has been implemented in several widely-used codes such as NASTRAN ${ }^{\mathrm{TM}}$, ANSYS ${ }^{\mathrm{TM}}$ and COMSOL ${ }^{\mathrm{TM}}$. This enables preparing simulations involving viscoelastic phenomena with relatively small changes in the material definitions compared to their standard elastic formulations.

Several studies or patents aiming at dimensioning and designing viscoelastic properties for vibrational and acoustic purposes have been presented in the literature. Jung and Aref [10] associated a honeycomb and viscoelastic materials for maximising the dissipation of energy in their structures. Their combined model was able to dissipate up to 2.5 times as much energy as the individual honeycomb or viscoelastic structure did. The authors outlined the shear resistance and energy dissipation opportunities their work presents, although the presented solution has not been applied to industrial devices such as rotating machines in operation.

De Lima et al. [11] designed a multi-objective optimisation procedure for finite-element models involving viscoelastic materials. The initial definition of the finite-element structure's dynamic equation is 
made with frequency-dependent hysteretic damping:

$$
\left[\mathscr{K}_{e}+G^{*}(\omega, T) \overline{\mathscr{K}_{v}}-\omega^{2} \mathscr{M}\right] \mathscr{Q}(\omega, T)=\mathscr{F}(\omega),
$$

where $\mathscr{K}_{e}$ is the stiffness matrix relative to the elastic materials, $G^{*}(\omega, T) \overline{\mathscr{K}}_{v}$ the complex viscoelastic stiffness matrix, $\omega=2 \cdot \pi \cdot f$ the circular frequency, $\mathscr{M}$ the mass matrix, $\mathscr{Q}(\omega, T)$ the vector of the harmonic generalized displacement magnitudes and $\mathscr{F}(\omega)$ the vector of the harmonic external loads. The optimisation algorithm the authors presented also included sub-structuring algorithms that successfully reduced the computation costs associated to the direct response resolution of Equation (1).

While comparing several viscoelastic material models, Trindade et al. [12] optimised the dimensions of a viscoelastic material layer in active-passive damping patches on a cantilever beam. Optimum dimensions increased the beam's modal damping factors up to $9 \%$ compared to the initial state.

Tuning the damping level in a structure may be achieved by temperature control, as proposed by Butaud et al. [13]. In this work, viscoelastic properties of a sandwich composite structure embedding a highly dissipative shape memory polymer are controlled, allowing drastic changes in the dynamical behaviour of the structure.

In addition to applied academic and industrial research studies, several patents have been applied in the field of vibration-damping viscoelastic materials. The works [14], [15] and [16] could be thought of, although the published documents lack of concrete applications on vibrating structures and external reports on their actual performances.

So far, this section has reviewed some of the state-of-the-art knowledge in modelling viscoelastic materials for finite-element simulations and concrete applications for reducing vibrations and noise levels in various structures. As for the acoustic behaviours of electric machines, some works have outlined the interesting potential of applying simulated electromagnetic excitations on structural finite-element models in order to compute representative noise levels radiated while functioning. In addition to the modelling guidelines proposed by Millithaler et al. [17, 18, 19], Schlensok et al. [20], Humbert et al. [21] and Dupont et al. [22] present multi-physical simulation methods including electromagnetic, structural and acoustic simulations for predicting the overall motor or powertrain noise emissions. Concerning design objectives though, to the authors' knowledge there are currently no published works detailing the optimisation of potting resins' viscoelastic properties for minimising electric motors' acoustic responses involving electromagnetic excitations.

To elaborate on these bases, this article will present a comprehensive methodology of resin viscoelastic property optimisation in order to minimise the vibration and noise of a switched-reluctance machine subjected to representative electromagnetic excitations. The method only involves finite-element simulations on common software programmes and is therefore easy to apply for other concrete industrial structures. This study intends to investigate new opportunities in the combined applications of viscoelasticity, electromagnetic excitations and noise, vibration and harshness (NVH) problems on electric machines. The following sections will begin with some basic notions about viscoelasticity and detail the applications on the studied electric machine afterwards. These applications are divided into two chronological stages: firstly the simulation of electromagnetic simulations and then the simulation of acoustic responses.

\section{Notions of viscoelasticity}

Polymers such as potting resins are usually described by viscoelastic properties [23]. Amongst other specificities, a viscoelastic material is characterised by a hysteretic stress-strain behaviour, which corresponds to an out-of-phase dynamic response of the material subjected to harmonic perturbations [24, 25, 
26]. The corresponding stress-strain relation involves the complex modulus $E^{*}$ which is classicaly written as

$$
E^{*}=E^{\prime}+\mathbf{i} \cdot E^{\prime \prime},
$$

introducing the storage modulus $E^{\prime}$ and the loss modulus $E^{\prime \prime}$, and where the ratio

$$
\frac{E^{\prime \prime}}{E^{\prime}}=\tan (\delta)
$$

is called loss factor and can be seen as a mechanical damping.

Although the dynamic behaviour of viscoelastic materials can be expressed as a dependency to the time, it will be rather seen as dependency to the frequency $f$ for the rest of this article, so that $f=\omega /(2 \cdot \pi)$. In addition to this, the dependency of viscoelastic properties to the temperature $T$ will be also accounted for [27].

Every viscoelastic material used in this study is considered homogeneous and isotropic, and thus defined by a Young's modulus, a shear modulus and a Poisson's ratio. In this study, Poisson's effects are assumed independent from temperature and frequency. This assumption seems reasonable as suggested in [12], [28], [11] and [7] and judging from the small discrepancies observed in the experimental identification presented in [29]. Therefore, the expression of a complex modulus $E^{*}(T, f)$ is related to a complex shear modulus $G^{*}(T, f)$ by the linear function inspired from isotropic elasticity [30]:

$$
G^{*}(T, f)=\frac{E^{*}(T, f)}{2 \cdot(1+v)},
$$

where $v$ is the material's Poisson's ratio. Equation (3) can thus be re-written in terms of shear moduli:

$$
G^{*}(T, f)=G^{\prime}(T, f) \cdot(1+\mathbf{i} \cdot \eta(T, f))
$$

where

$$
\eta=\tan (\delta)
$$

\section{Experimental analysis on a resin sample}

In order to understand the dynamic behaviour of a given potting resin used for switched-reluctance machines and namely its dependencies to temperature and frequency, an experimental dynamic-mechanical analysis (DMA) is performed on a sample of this material. This consists in applying a harmonic load to the sample and measuring its response, in order to determine $E^{\prime}, E^{\prime \prime}$ and $\tan (\delta)$.

Concretely, a sample of a resin called P3 is created (developed and supplied by the company Von Roll) with a shape of a 70-mm-long rod of 2-mm $\times 4-\mathrm{mm}$ rectangular section, and is subjected to harmonic strains in tension along its length (there also exist other DMA methods such as bending, but they will not be presented here). The test is repeated between $-39^{\circ} \mathrm{C}$ and $101^{\circ} \mathrm{C}$, and between $0.1 \mathrm{~Hz}$ and $10 \mathrm{~Hz}$. The resulting curves are plotted in Figure 2.

As it can be seen in Figure 2, important variations are observed at higher temperatures. Each curve $\eta(T)$ reaches a maximum between $80^{\circ} \mathrm{C}$ and $100^{\circ} \mathrm{C}$. Although there exist more specific definitions for this entity, the temperature at which the loss factor reaches a maximum will be seen as the frequencydependent glass transition temperature $T_{G}(f)$ [31]. In a range of about $T_{G} \pm 10^{\circ} \mathrm{C}$, it can be seen that the values of the material's mechanical damping are above 0.5 . 

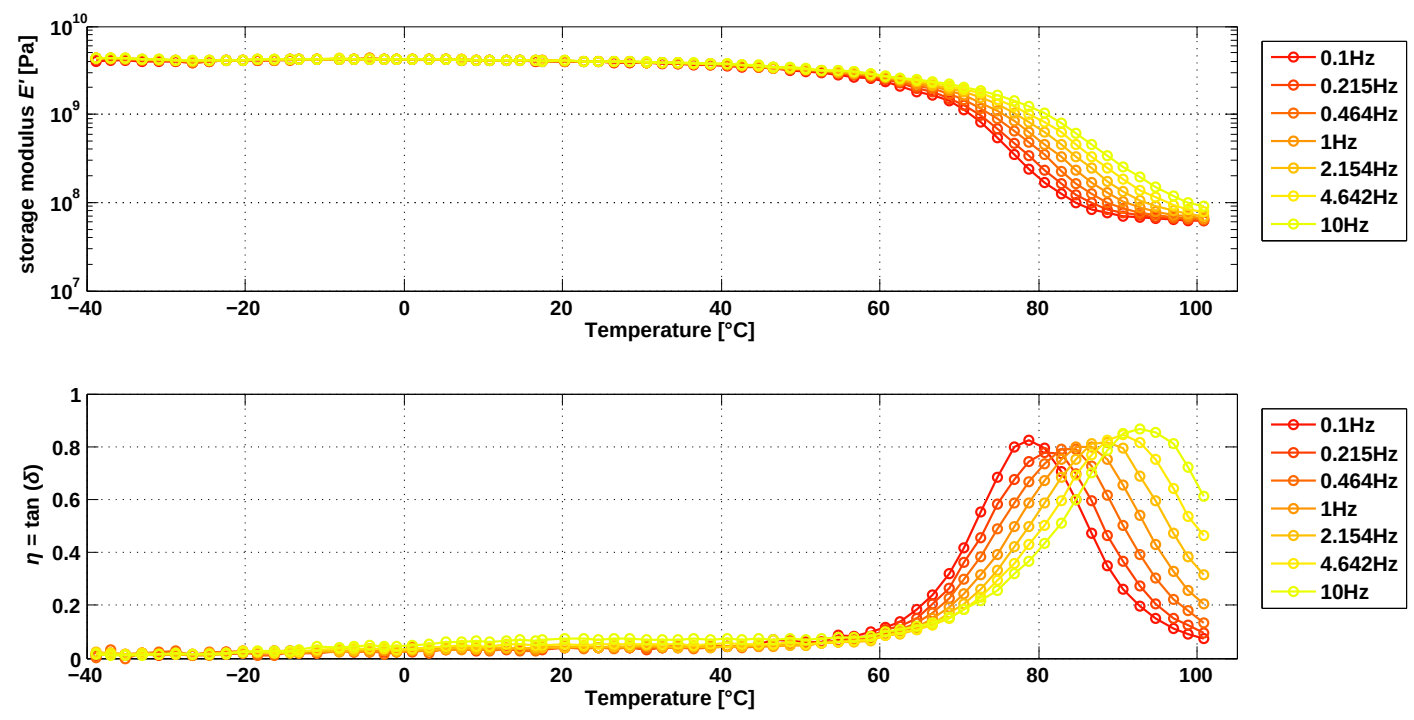

Figure 2: DMA results for the storage modulus and loss factor changes in P3 sample with temperature and frequency

As outlined in [32], despite the important amount of information the DMA results give, the definitions are relatively limited in terms of frequency. This is due to practical reasons, such as the analyser's excitation limitations. However, using algorithms based on the time-temperature superposition principle (see e.g. [33], [34]) enables to compute so-called master-curves over a larger frequency range. The mastercurves at $81^{\circ} \mathrm{C}$ computed from the resin P3's DMA measurements are plotted in 3 .

Finally, considering a symmetry about the median frequency value, the similarities in the shapes of the loss factor curves in Figures 2 and 3 is an interesting aspect to bring under the spotlight. Indeed, whereas in Figure 2, the high-damping range $(\eta>0.4)$ was about $20^{\circ} \mathrm{C}$-wide, Figure 2 shows that these values span over more than three decades in terms of frequency. It is this specificity that this study aims at taking advantage of.

\section{Analytical modelling of viscoelastic properties}

As it has been stated earlier, the objective of this article is to determine optimum viscoelastic properties for a stator's potting resin. For convenience in this tuning process, these properties need to be described by easily-controllable parameters. This is why analytical models are used to approximate the polymer's real behaviour. For consistency with the electromagnetic excitations simulated as frequency spectra, viscoelastic effects will be modelled with frequency-dependent moduli at fixed temperatures, therefore corresponding to the above-presented master-curves.

\subsection{Zener model}

Several analytical formulations exist to describe the frequency dependency of viscoelastic moduli [29]. The fractional derivative Zener model [35] is particularly convenient to use in this study as it is generated from four easily-identified input parameters, namely the static elastic modulus

$$
E_{0}=\lim _{f \rightarrow 0} E^{\prime}(f)
$$



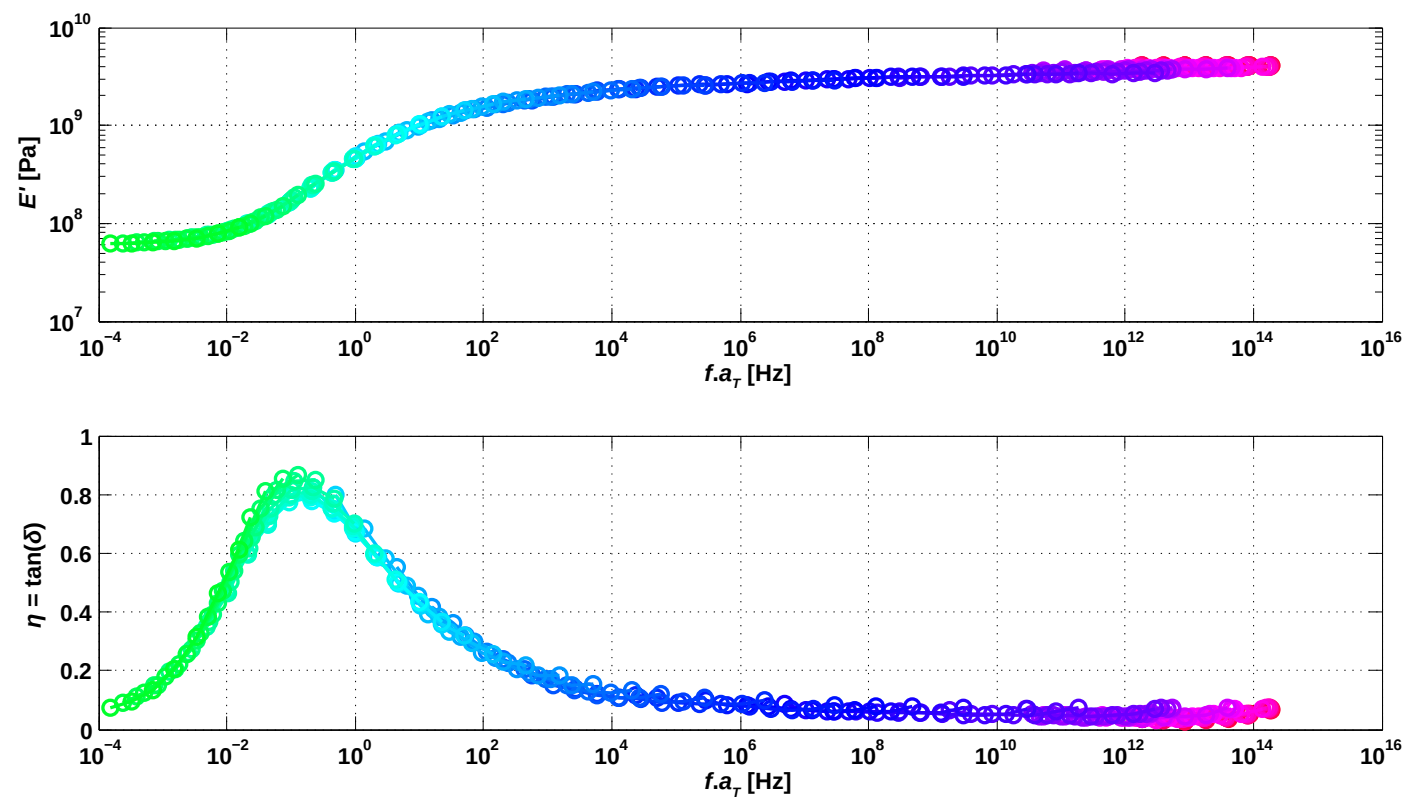

Figure 3: Master-curves for resin $\mathrm{P} 3$ with $T_{0}=81^{\circ} \mathrm{C}$

the high-frequency modulus

$$
E_{\infty}=\lim _{f \rightarrow+\infty} E^{\prime}(f)
$$

the maximum loss factor

$$
\eta_{\max }=\max _{f} \eta(f)
$$

and the corresponding frequency

$$
f_{\eta_{-} \max }=\left.f\right|_{\eta(f)=\eta_{\max }} .
$$

For convenience, $f_{\eta_{-} \max }$ will be referred to as "master-frequency" in this entire article. The determination of these input parameters is illustrated in the (fictive) master-curve example in Figure 4.

Then, the order of fractional derivative $\alpha$ and the material's relaxation time $\tau$ are determined with the expressions [28, 35]

$$
\alpha=\frac{2}{\pi} \cdot \arcsin \left(\eta_{\text {max }} \cdot\left(E_{\infty}-E_{0}\right) \cdot \frac{2 \cdot \sqrt{E_{\infty} \cdot E_{0}}+\left(E_{\infty}+E_{0}\right) \cdot \sqrt{1+\eta_{\text {max }}^{2}}}{\eta_{\text {max }}^{2} \cdot\left(E_{\infty}+E_{0}\right)^{2}+\left(E_{\infty}-E_{0}\right)^{2}}\right)
$$

and

$$
\tau=\frac{1}{2 \cdot \pi \cdot f_{\eta \_} \max } \cdot\left(\frac{E_{0}}{E_{\infty}}\right)^{\frac{1}{2 \cdot \alpha}}
$$

with the aid of which the complex Young's modulus is constituted:

$$
E^{*}(f)=\frac{E_{0}+E_{\infty} \cdot(\mathbf{i} \cdot f \cdot \tau)^{\alpha}}{1+(\mathbf{i} \cdot f \cdot \tau)^{\alpha}}
$$



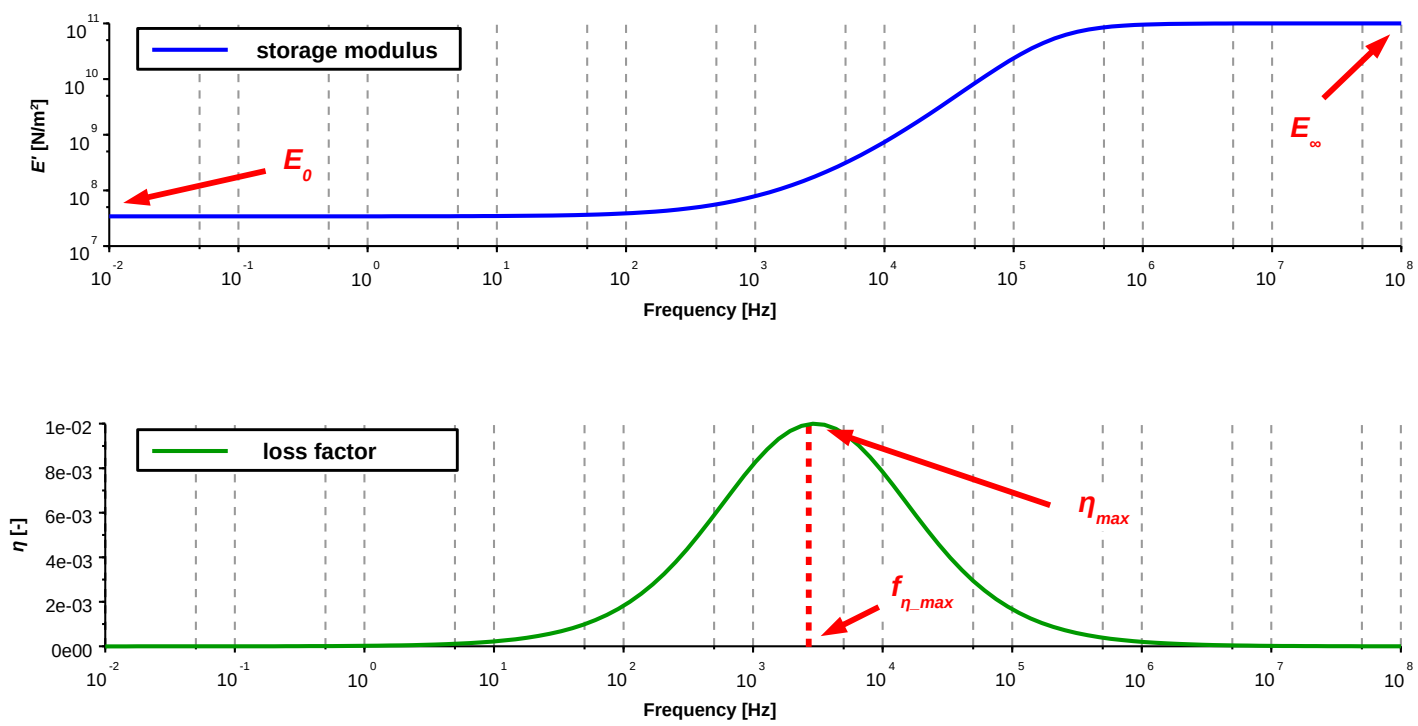

Figure 4: Input parameters for the Zener model

Finally, using the same expressions as shown in Section 2 enables to compute the associated storage and loss moduli as well as the loss factor. Considering shear moduli instead of Young's moduli with the Zener model is applied in an identical way as long as Equation (5) holds.

In order to model resin P3 at the temperature $T_{0}=81{ }^{\circ} \mathrm{C}$, the Zener input values are identified from the results plotted in Figure 3: $E_{0}=6 \cdot 10^{7} \mathrm{~Pa}, E_{\infty}=4 \cdot 10^{9} \mathrm{~Pa}, \eta_{\max }=0.8$ and $f_{\eta_{-} \max }=10^{-1} \mathrm{~Hz}$. The comparison between the master-curves of resin $\mathrm{P} 3$ at the temperature $T_{0}=81^{\circ} \mathrm{C}$ and the corresponding analytical curves $E^{\prime}(f)$ and $\eta(f)$ is shown in Figure (5).

The shapes of the analytical and the experimental curves are reasonably similar, although there exist some discrepancies. Amongst other aspects, it can be seen that Zener's loss modulus curve $\eta(f)$ is symmetric about $f_{\eta_{-} \max }$, which is not clearly the case for the corresponding master-curve. Considering other analytical laws such as the models presented in [5] or [29] could improve the representativeness. However, the Zener parameters $E_{0}, E_{\infty}, \eta_{\max }$ and $f_{\eta_{-} \max }$ are directly related with the concrete properties this study aims at designing in a polymer. Judging from the pre-processing simplicity and the control over convenient physical parameters it presents, the Zener model is therefore preferred.

\subsection{Extrapolation of frequency-temperature models}

As stated at the end of Section 3, a master-curve can be seen as the extended isothermal behaviour of the material at the reference temperature chosen for its computation. In addition to the practical approach detailed in Section 3 for computing frequency shift factors, it can be noted that several analytic laws have been proposed for modelling the dependency of $a_{T}$ towards $T$. Amongst the most common, the Arrhenius law can be seen as a linear function of $\ln \left(a_{T}\right)$ with respect to $1 / T$, whereas the Williams-Landel-Ferry (WLF) law is a linear function of $1 / \log \left(a_{T}\right)$ with respect to $1 /\left(T-T_{0}\right)$ [36]. In detail, numerical evaluations enable to approximate Arrhenius and WLF laws for $T_{0}=81{ }^{\circ} \mathrm{C}$ with the respective linear functions:

$$
\ln \left(a_{T}\right)=8.489 \cdot 10^{4} \cdot \frac{1}{T}-2.387 \cdot 10^{2}
$$

and 

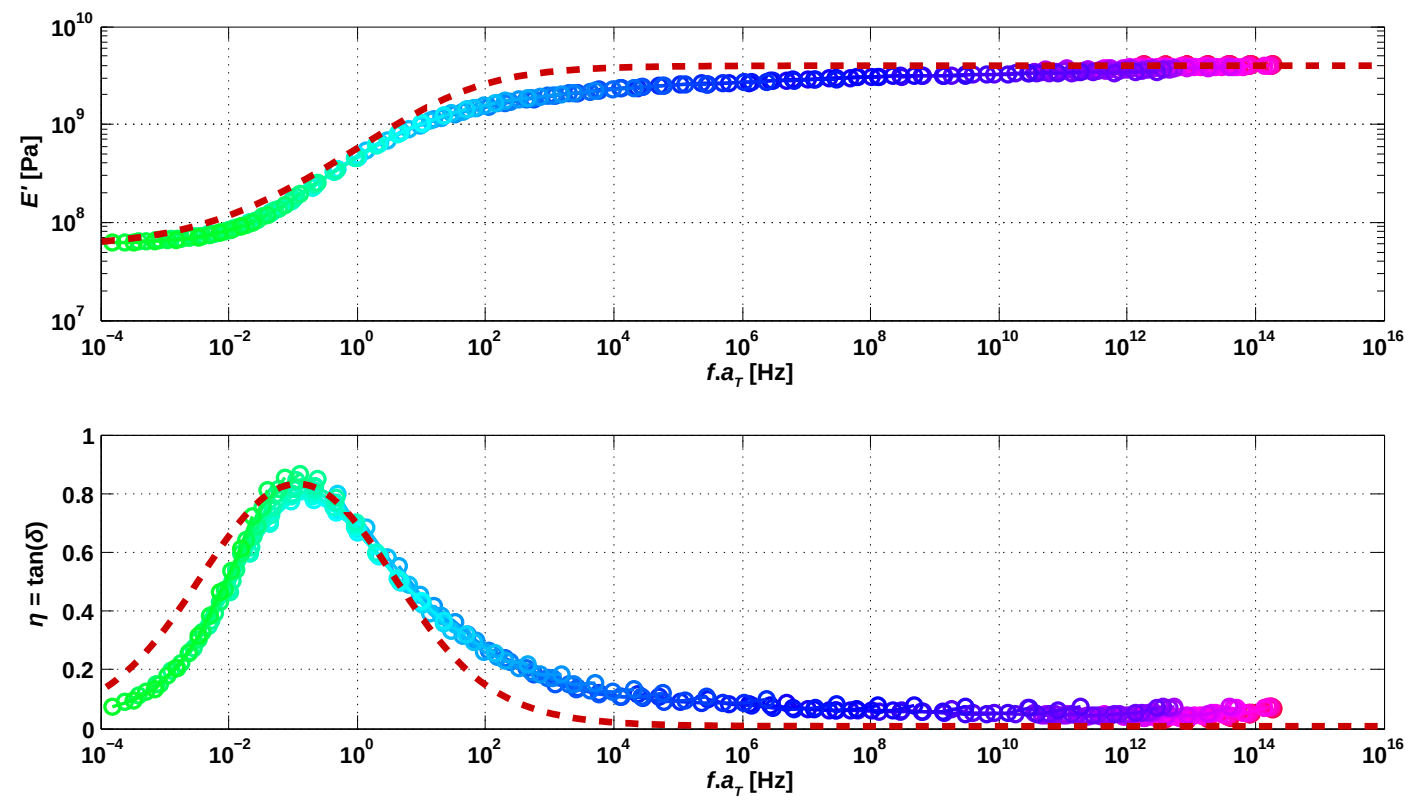

Figure 5: Master-curves for resin P3 with $T_{0}=81^{\circ} \mathrm{C}$ (solid lines and circles) and corresponding Zener models (red dashed lines)

$$
\frac{1}{\log \left(a_{T}\right)}=-5.740 \cdot \frac{1}{T-T_{0}}+9.709 \cdot 10^{-2},
$$

where $\ln$ is the natural logarithm (base $e$ ) and log the common logarithm (base 10).

However, such laws are only valid in certain temperature ranges and possibly depend on experimentallybased constants. In order to overcome these limitations and approximate the behaviour of the resin in wide ranges of both temperatures and frequencies, the evolution of the "master-frequency" $f_{\eta_{-} \max }$ (see Equation (11)) is analysed for a few reference temperatures $T_{0}$. A polynomial function is computed to fit the values (minimisation of least squares), as plotted in Figure 6.

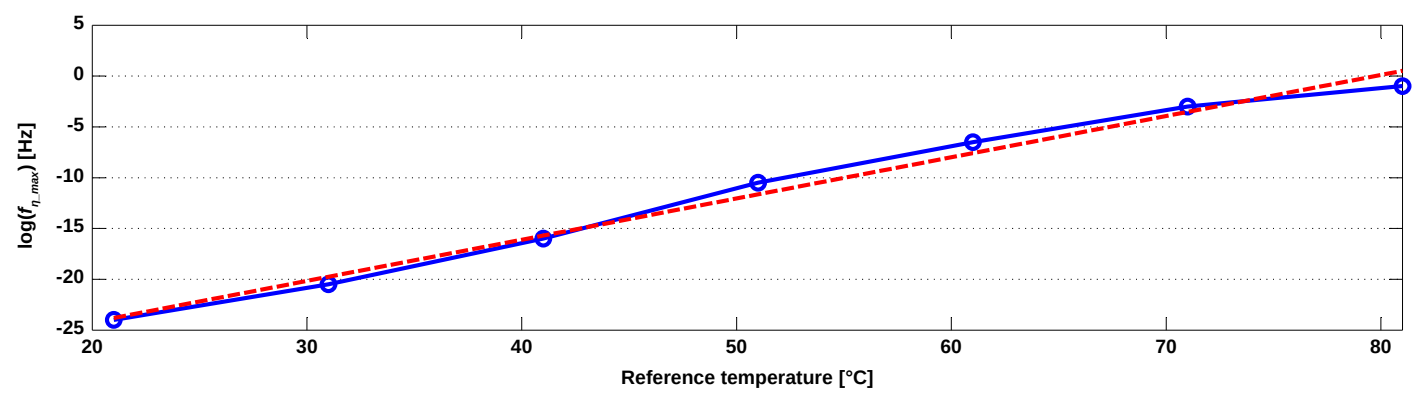

Figure 6: Measured master-frequencies (blue circles) and fitting polynomial curve (red dashed line)

The following first-order polynomial function is identified from the values:

$$
\log \left(f_{\eta_{\_} \max }\right)=0.4054 \cdot T_{0}-32.316 .
$$


The coefficient of determination $\mathscr{D}$ associated to this fit is computed with the expression

$$
\mathscr{D}=1-\frac{\sum_{i \in \mathscr{T}_{\text {ref }}}\left(\log \left(f_{i}^{\{m\}}\right)-\log \left(f_{i}^{\{c\}}\right)\right)^{2}}{\sum_{i \in \mathscr{T}_{\text {ref }}}\left(\log \left(f_{i}^{\{c\}}\right)\right)^{2}},
$$

where $\mathscr{T}_{\text {ref }}$ is the set of reference temperatures, $f_{i}^{\{m\}}$ are the measured master-frequencies and $f_{i}^{\{c\}}$ the frequencies calculated with Equation (17). The value $\mathscr{D}=99.59 \%$ confirms the good agreement between the experimental and the analytical evolutions of master-frequencies with respect to reference temperatures. Extrapolating the values of $f_{\eta_{-} \max }$ with relation (17) and using Zener models enables to predict the viscoelastic behaviour of the resin in wide ranges of temperatures $T$ and frequencies $f$. The evolutions of the storage modulus $E^{\prime}$ and the loss factor $\eta$ with respect to $T$ and $f$ are plotted in Figure 7 .
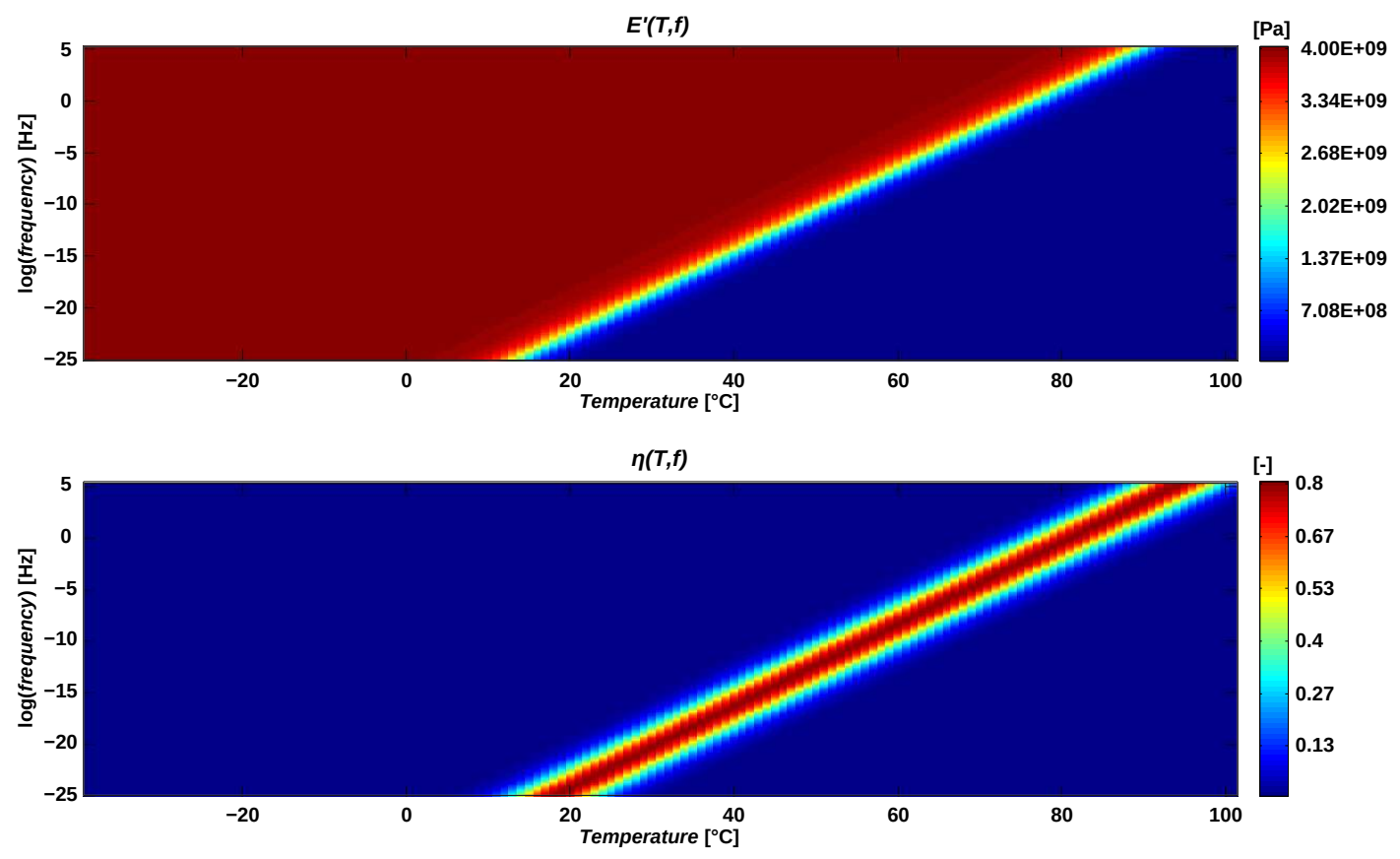

Figure 7: Graphical representations of $E^{\prime}(T, f)$ and $\eta(T, f)$

\section{Electromagnetic excitations}

The device studied in this article is a "12/8" (12-toothed stator, 8-toothed rotor) switched-reluctance motor designed for automotive $100 \%$-electric traction vehicles. The number of teeth are referred to as $Z_{S}$ for the stator and $Z_{R}$ for the rotor. A 2-D mesh is designed for electromagnetic finite element analyses, and is shown in Figure 8. It is composed of 221,985 nodes and 105,454 elements. Symmetries and antisymmetries could have been used to reduce the size of the problem.

In a switched-reluctance machine, the stator windings are powered sequentially so that the magnetic flux circulating from the stator to the rotor creates torque while aligning the rotor teeth with the stator poles. On the illustration shown on Figure 9, the winding phase "3" (right-hand side) is powered directly 


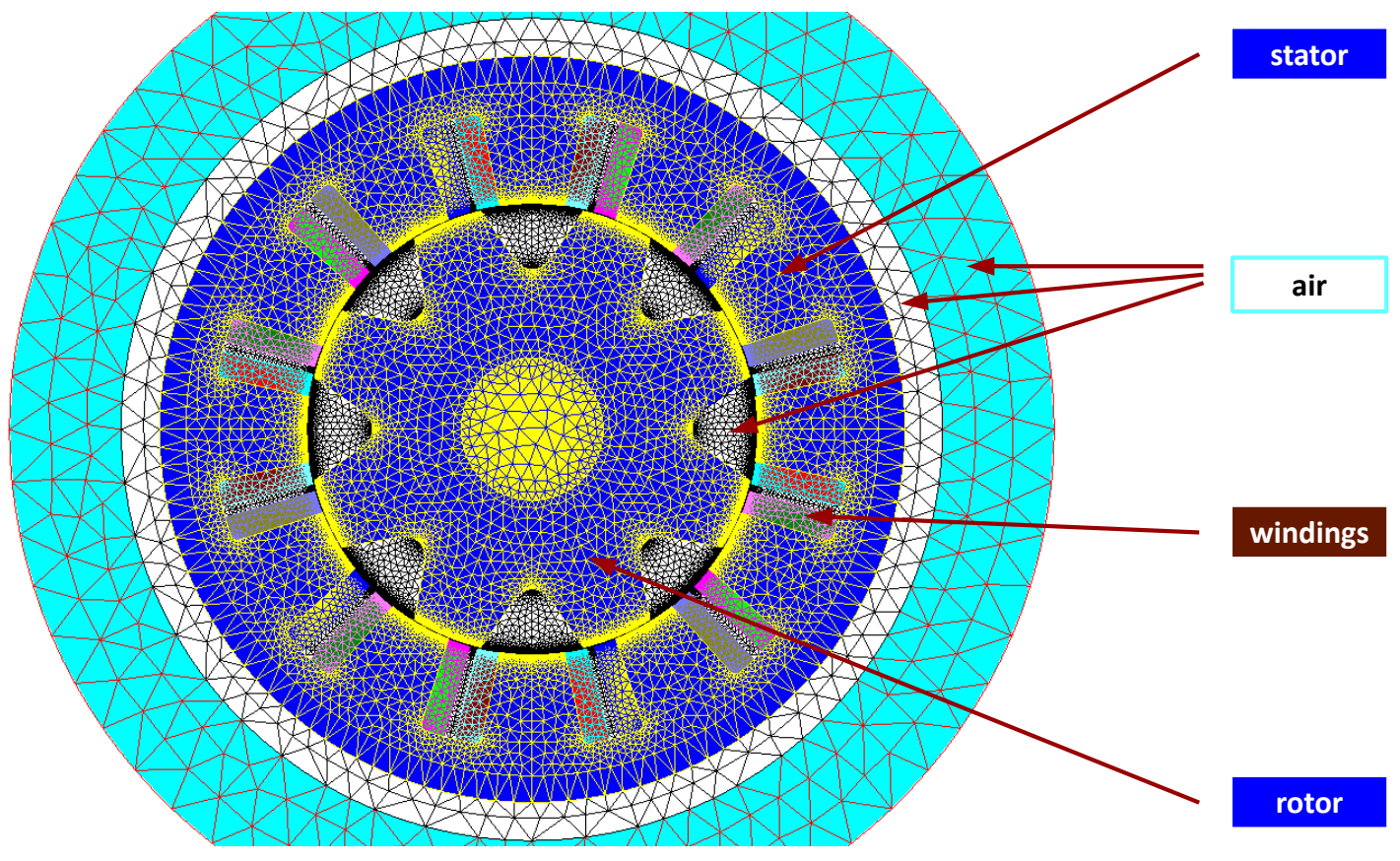

Figure 8: Finite-element mesh for electromagnetic simulations (Cedrat Flux2D ${ }^{\mathrm{TM}}$ )

after phase "1" (left-hand side): by minimising the distance in the air, the circulation of the flux (thin lines) creates torque, until the next pair of coils is powered instead and the rotor is spun anew.
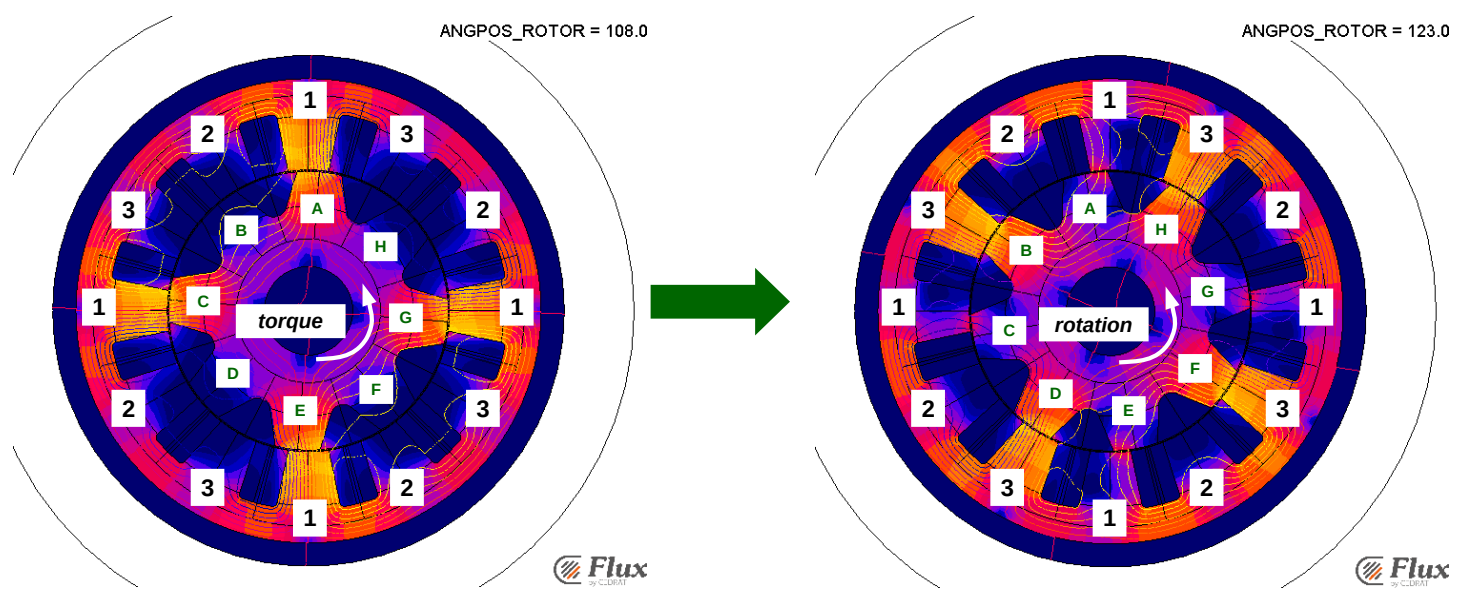

Figure 9: Flux density maps in the SRM

For each of the rotor speeds $\mathscr{N}_{r}$ of $500,1000,2000,3000, \ldots$ and 12000 revolutions per minute $(\mathrm{rpm})$, a simulation is performed with the electromagnetic finite-element solver Cedrat Flux2 $\mathrm{D}^{\mathrm{TM}}$. The input voltage $U_{i}$ is controlled in order to impose a constant global magnetic flux $\Phi_{g}$ in the motor (proportional to the ratio $U_{i} / \mathscr{N}_{r}$ ), so that $U_{i}$ does not exceed the battery voltage $U_{b}=400 \mathrm{~V}$ in the speed range [500; $12000 \mathrm{rpm}]$. Therefore, the input voltage for each simulation is computed from the corresponding rotor speed with

$$
U_{i}=U_{b} \cdot \frac{\mathscr{N}_{r}}{12000}
$$


Finally, flux density values $B\left(\theta_{s}, \theta_{r}\right)$ are computed at the motor's air gap for each rotor position $\theta_{r}$. The virtual sensors where flux densities are computed are localised with their angular values $\theta_{s}$, and are illustrated in Figure 10. This enables to determine Maxwell stresses $P_{n}$ and $P_{t}$ with the expressions [37, 38]

$$
P_{n}\left(\theta_{s}, \theta_{r}\right)=\frac{\left[B_{n}\left(\theta_{s}, \theta_{r}\right)\right]^{2}-\left[B_{t}\left(\theta_{s}, \theta_{r}\right)\right]^{2}}{2 \cdot \mu_{0}}
$$

and

$$
P_{t}\left(\theta_{s}, \theta_{r}\right)=\frac{B_{n}\left(\theta_{s}, \theta_{r}\right) \cdot B_{t}\left(\theta_{s}, \theta_{r}\right)}{\mu_{0}},
$$

where indices $n$ and $t$ respectively refer to normal (radial) and tangential components, and where $\mu_{0}$ is the magnetic permeability of vacuum. Maps of the radial and tangential Maxwell stress distributions are shown in Figure 11, where angle values are localised in a positive trigonometric (i.e. anti-clockwise) system about axis $+z$.

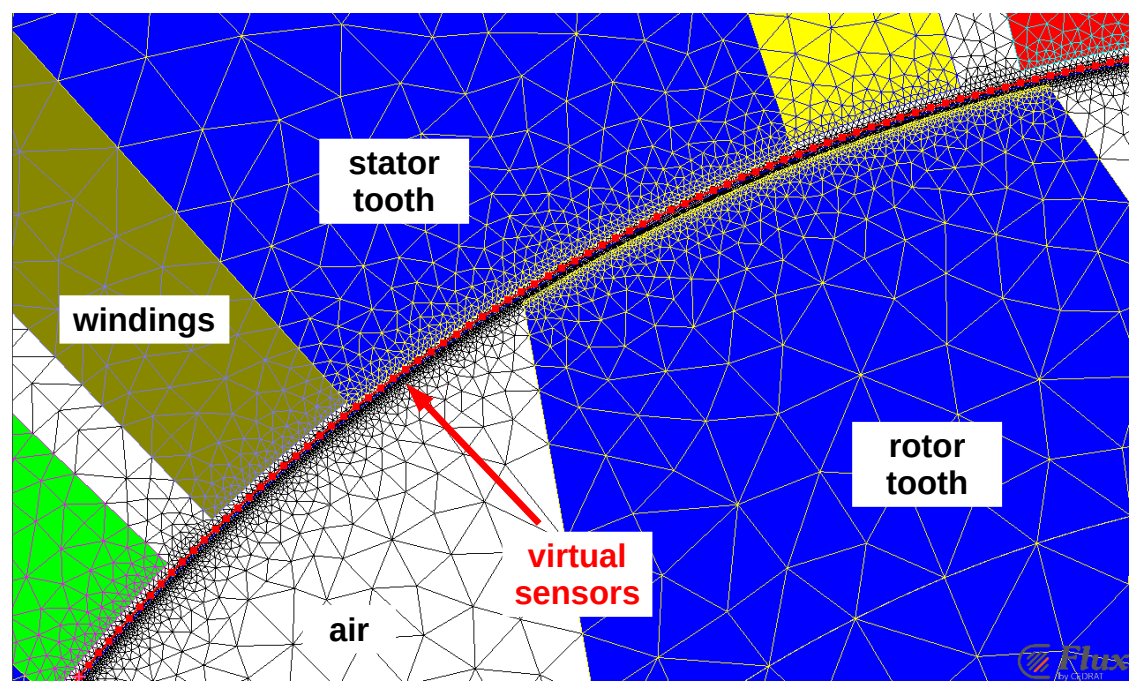

Figure 10: Virtual sensors in the air gap
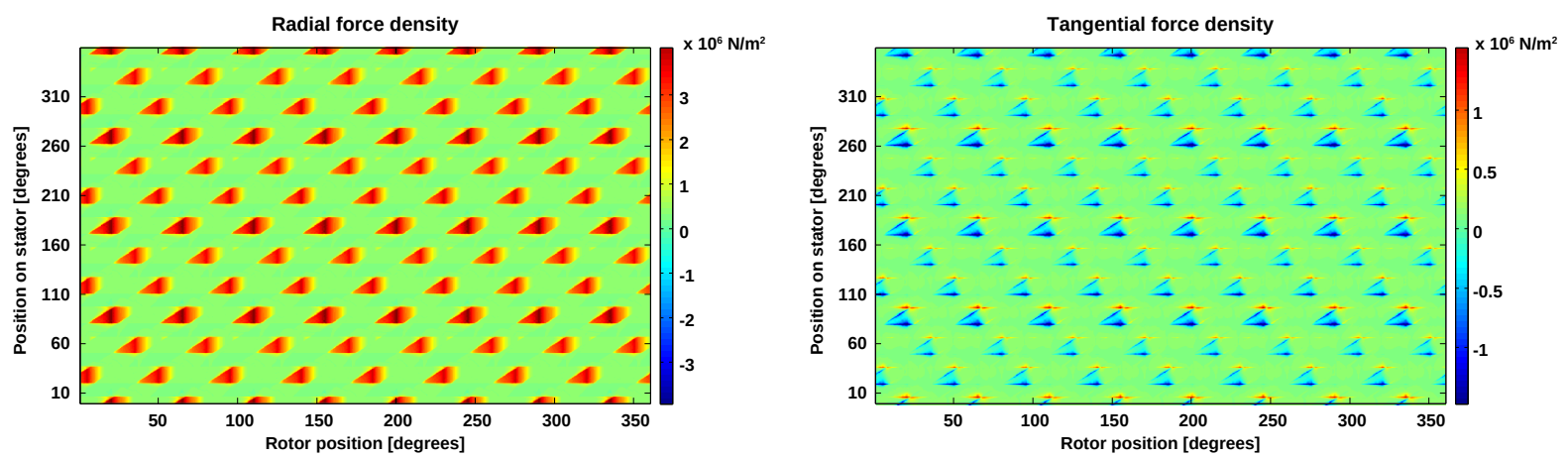

Figure 11: Spatial distributions of Maxwell stresses

The distribution maps clearly show that radial Maxwell stress components are significantly greater than tangential contributions, which is consistent with the remarks outlined in [39] and in [21]. Also, the 
rotor circumference clearly exhibits 8 magnitude maxima, and the stator 12 . These numbers expectedly correspond to the numbers of teeth $Z_{R}$ and $Z_{S}$, respectively.

The next step consists in analysing spectral representations of these excitation maps. Assimilating $\mathscr{N}_{r}$ to a circular frequency $\omega_{r}=2 \cdot \pi \cdot \mathscr{N}_{r} / 60$ and $\theta_{r}$ to the time $t=\theta_{r} / \omega_{r}$, and computing Fourier transforms of $P_{n}$ and $P_{t}$ about both variables yields the spectral matrices $\mathscr{P}_{n}$ and $\mathscr{P}_{t}$, that describe the electromagnetic excitations in terms of spatial orders $m_{e}$ and frequencies $f_{e}$. A graphical representation of matrix $\mathscr{P}_{n}$ is shown in Figure 12, where engine orders $k$ (referred to as " $\mathbf{H}_{k}$ ") are multiples of the engine's fundamental frequency and are defined by the expression

$$
k=\frac{2 \cdot \pi \cdot f_{e}}{\omega_{r}} .
$$

Therefore, the engine order $\mathbf{H}_{0}$ is not considered for dynamic analyses.

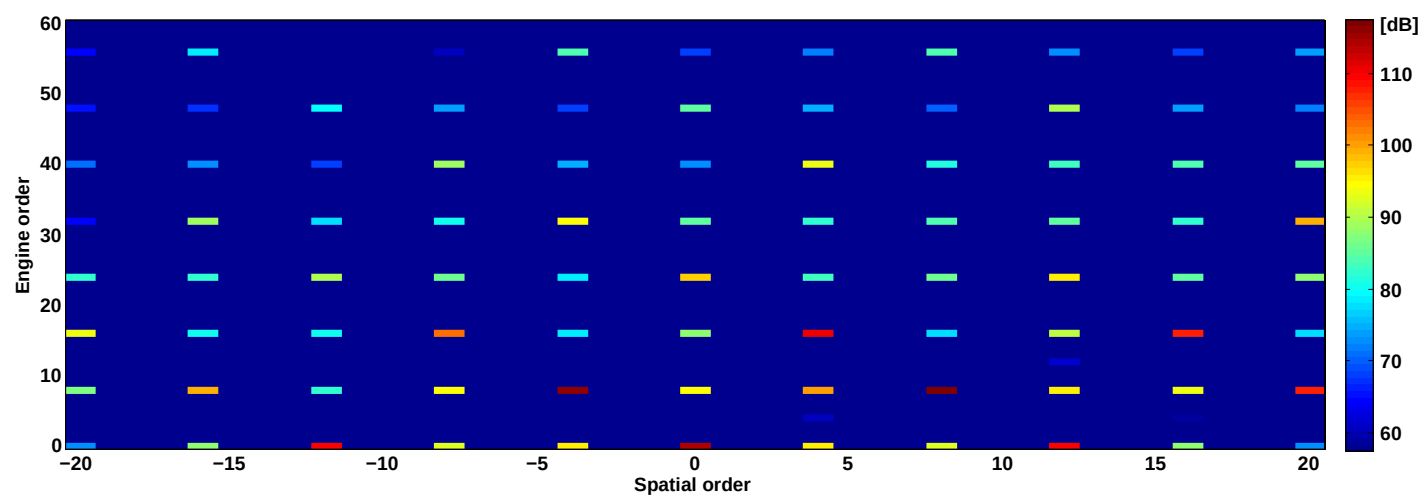

Figure 12: Engine order - spatial order representation of the radial excitation (for $\mathscr{N}_{r}=1,000 \mathrm{rpm}$ )

Several observations can be made from the representation in Figure 12:

- The highest excitation magnitudes are found at low engine orders.

- The excitation magnitudes are negligible if the engine order is not a multiple of eight. This is consistent with the facts that the highest Maxwell stress magnitudes are observed at the conjunctions of stator and rotor teeth, and that conjunctions occur eight times (as $Z_{R}=8$ ) per rotor revolution at each stator tooth (see Figure 11). Concretely, excitation spectra of higher engine orders are harmonics of the fundamental $\mathbf{H}_{8}$.

- In a similar way, the magnitudes are negligible if the spatial order is not a multiple of four. This corresponds to the greatest common divisor of $Z_{S}$ and $Z_{R}$, which is a consequence of the Fourier transforms. In practice, the spatial order of an excitation distribution corresponds to the number of magnitude maxima in the air gap circumference. Due to the complex nature of the excitations, magnitude maxima are displaced with increasing angle values at the air gap circumference while the stress phases are increased. In other terms, observing the excitation in a trigonometric view for a given frequency and a given positive spatial order shows a positive rotation of magnitude maxima. This orientation is reversed for negative spatial orders.

This representation has a similar shape for other values of $\mathscr{N}_{r}$. The global radial force resultant and tangential force resultant (i.e. torque) are integrated over the entire air gap's surface, and are plotted in 
Figure 13. It can be clearly seen that the global radial resultant is almost constant in the speed range $[500 ; 12,000 \mathrm{rpm}]$.
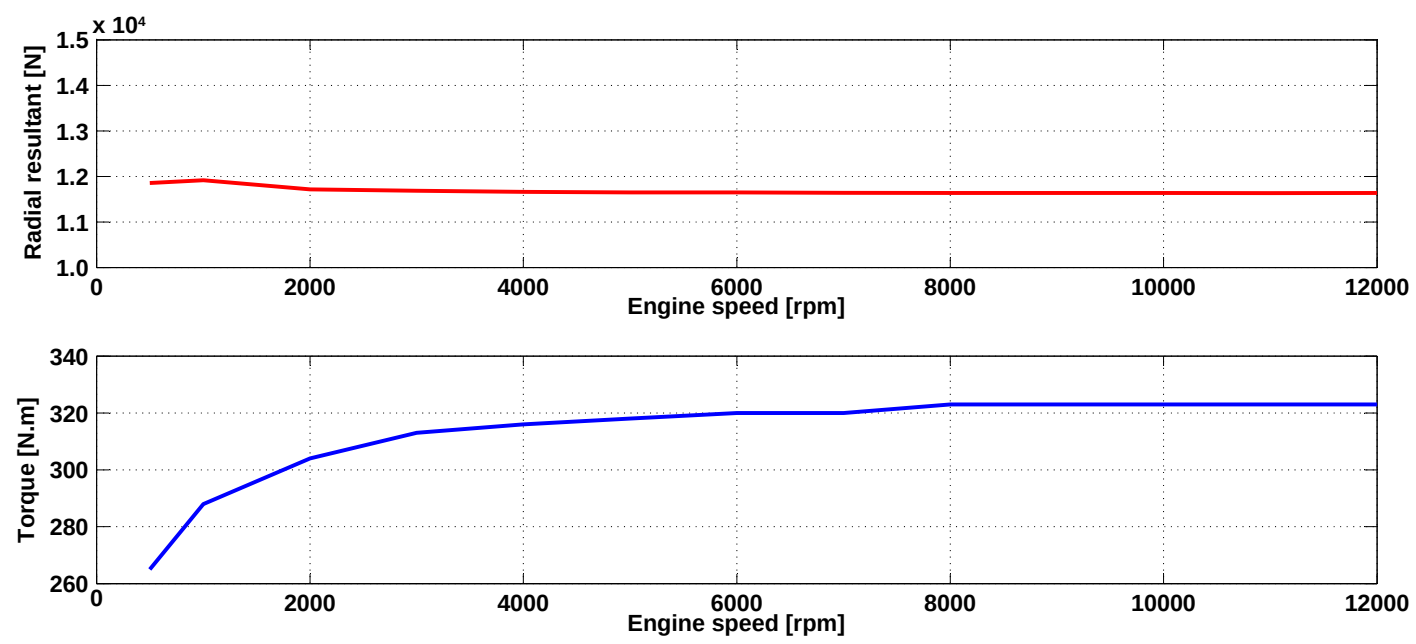

Figure 13: Global radial and tangential resultant forces at the air gap

In the following stages of this study, dynamic responses will be simulated for rotor speeds between 250 and 12,000 rpm with steps of $250 \mathrm{rpm}$ (thus 48 speed cases), where engine orders above $\mathbf{H}_{56}$ as well as spatial orders beyond the range $[-20 ;+20]$ will be discarded. However, generating 48 excitation spectra represents an important amount of data and therefore significantly long simulations. This is why the extraction of excitation spectra according to specific engine orders is preferred. Therefore, only the excitation spectra $\mathbf{H}_{8}, \mathbf{H}_{16}, \mathbf{H}_{24}, \mathbf{H}_{32}, \mathbf{H}_{40}, \mathbf{H}_{48}$ and $\mathbf{H}_{56}$ are considered for the dynamic response simulations. For a given excitation spectrum, the structure's deformations can be separated into independent responses to each of spatial force harmonics, according to the superposition principle. Eventually, Maxwell stresses are interpolated from neighbouring values if no electromagnetic simulation has been performed at the given rotor speed.

\section{Structural dynamic response}

Now that the electromagnetic excitations are processed, the dynamic response of the structure can be simulated. At this stage, modelling the rotor is no longer necessary. The entire stator is meshed in three dimensions, with 81,719 nodes and 58,634 solid elements. The main properties of the finite-element model are detailed as following:

- outer diameter: $245 \mathrm{~mm}$;

- inner diameter (at stator teeth): $127.2 \mathrm{~mm}$;

- air gap length (about direction z): $154 \mathrm{~mm}$;

- mass: $34.1 \mathrm{~kg}$.

The nodal coordinates as well as the material properties are expressed in the cylindrical coordinate system $\{r, \theta, z\}$, where $z$ is the stator's revolution axis. In order to stabilise the structure, four additional node-to-ground stiffness elements are connected to the frame at each of the stator's end faces. No 
damping is considered in these elements, while their stiffness values are $1,000 \mathrm{~N} \cdot \mathrm{m}^{-1}$ in every direction (sufficiently high for an effective stabilisation and still negligibly low for not perturbing the structure's overall behaviour). An illustration of the entire mesh is given in Figure 14.

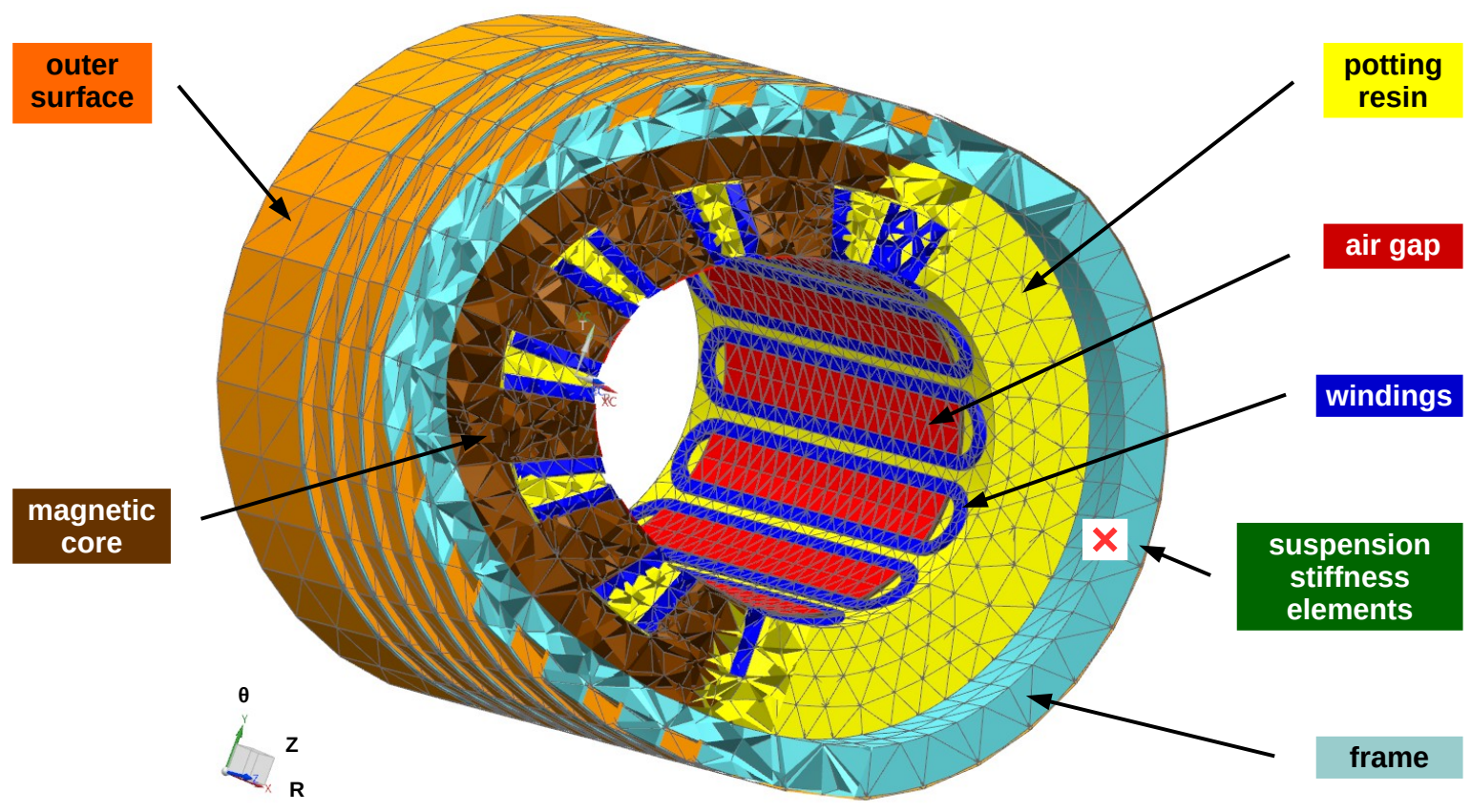

Figure 14: Cutaway of the stator's mesh

As shown in Figure 14, the air gap is identified by the core teeth's inner faces. As a prerequisite for the simulation output detailed in Subsection 6.2, the frame's lateral surface is coated with shell elements ("outer surface" in Figure 14). The associated material has negligible stiffness and thickness properties in order not to alter the overall mechanical behaviour.

\subsection{Modal basis}

Before computing dynamic responses, comparing the structure's modal behaviour to the excitation spectra's spatial distributions is a good indicator to anticipate resonance phenomena. In the case of a frequency-independent non-dissipative system (thus neglecting damping effects), the $i$-th eigenvalue can be described with the expression [40]

$$
\left(\mathscr{K}-\omega_{i}^{2} \mathscr{M}\right) \phi_{i}=\mathbf{0},
$$

where $\mathscr{K}$ (resp. $\mathscr{M}$ ) is the entire system's stiffness (resp. mass) matrix. The associated natural frequency $f_{i}=\omega_{i} / 2 \pi$ and deformed shape $\phi_{i}$ constitute the structure's $i$-th mode (real values in this case). With the aid of Equation 22, associating the excitation spectra $\mathbf{H}_{8}$ to $\mathbf{H}_{56}$ and the speed range [250; 12,000 rpm] corresponds to the frequency range $[66.7 ; 11,200 \mathrm{~Hz}]$. Therefore, the modes of the reference structure are computed up to $11,200 \mathrm{~Hz}$. The reference structure is composed of frequency- and temperature-independent material properties. In particular, the resin is modelled with constant elastic properties corresponding to the measured low-temperature Young's modulus $E=4 \cdot 10^{9} \mathrm{~Pa}$ (see Figure 2) and Poisson's ratio $v=0.38$.

In this case, the reference modal basis contains 77 modes, the first 6 of which (below $10 \mathrm{~Hz}$ ) describe the rigid-body suspension of the structure on the node-to-ground stiffness elements. For convenience, 
radial mode shapes are labelled with " $(m, n)$ ", where $m$ is the spatial order and $n$ the number of undeformed nodal circles about the stator length. Analysing the entire modal basis indicates that the highest spatial order $m$ (number of lobes on the stator's circumference) in the considered frequency range is 5 , for a mode at $11,729 \mathrm{~Hz}$. As resonance occurs when both the spatial order and the frequency of the excitation coincide with the mode, the only modes to focus on are thus of spatial orders 0 and 4 , and whose deformed shapes are symmetric about the stator length (even values for $n$ ):

- "breathing" mode $(0,0)$ at $6,295 \mathrm{~Hz}$,

- "ovalisation" mode $(4,0)$ at 7,090 Hz,

- mode $(0,2)$ at $7,416 \mathrm{~Hz}$,

- mode $(4,2)$ at $8,747 \mathrm{~Hz}$.

The modes are referred to as "critical", and are illustrated in Figure 15.

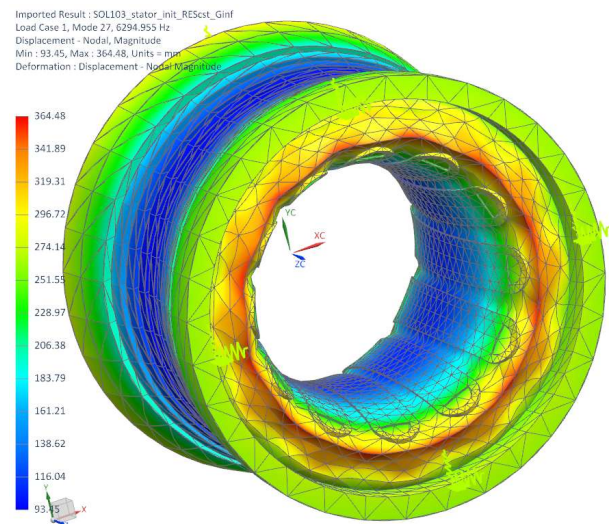

(a): mode $(0,0)-6,295 \mathrm{~Hz}$

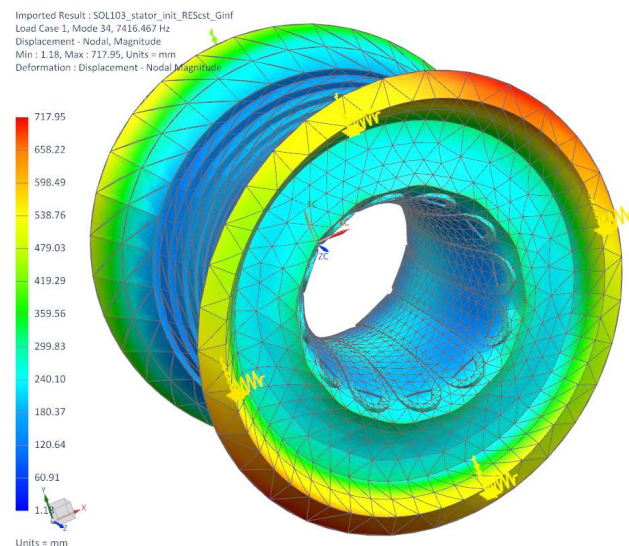

(c): mode $(0,2)-7,416 \mathrm{~Hz}$

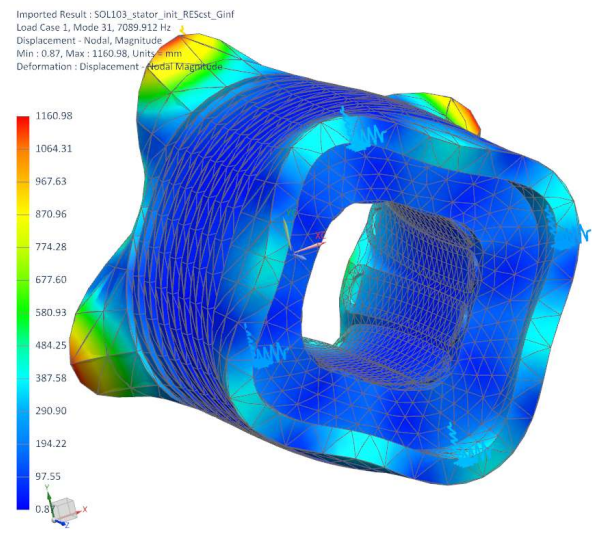

(b): mode $(4,0)-7,090 \mathrm{~Hz}$

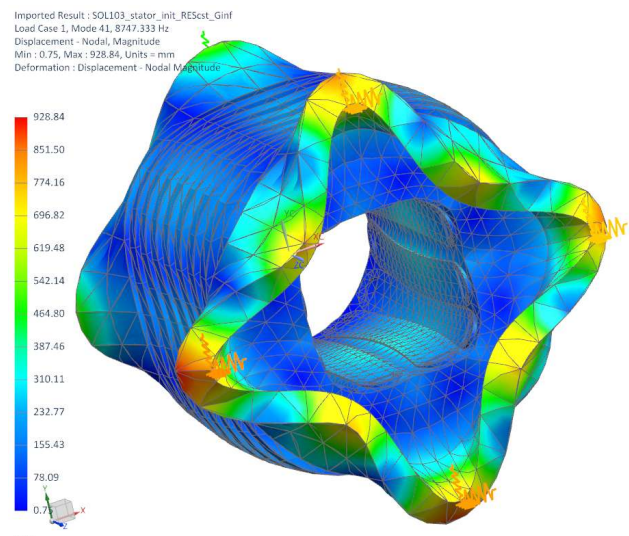

(d): mode $(4,2)-8,747 \mathrm{~Hz}$

Figure 15: Critical modes below 12,000 Hz 


\subsection{Simulation details}

At the opposite of the above-presented modal basis computation, the response simulations involve the electromagnetic excitations detailed in Section 5, which are projected onto the air gap nodes (see Figure 14). In order to estimate the acoustic emission associated to a given simulation, the Equivalent Radiated Power (ERP) responses are computed at the stator's "outer surface" panel. This type of output is commonly used in the automotive industry [41] for its direct estimation of radiated power without need to be post-processed through an acoustic simulation tool. Such outputs are only enabled on shell and plate elements (hence the choice of coating the frame's lateral face), but this choice has negligible impact on the global structure's behaviour and is more cost-effective than standard computations of normal velocities at nodes. ERP data are summed over all the nodes and elements of the panel with the following global expression [41]:

$$
E R P(f)=\rho_{\text {air }} \cdot c_{\text {air }} \cdot \sum_{j \in \text { panel }} V_{j, n}^{2}(f) \cdot S_{j}
$$

where $\rho_{\text {air }}$ is the density of air, $c_{\text {air }}$ the speed of sound in air, $V_{j, n}(f)$ the normal (i.e. radial) velocities at element $j$ and frequency $f$ and $S_{j}$ surface of element $j$.

The temperature is considered fixed at $T_{0}=81^{\circ} \mathrm{C}$, so that only frequency-dependent phenomena are modelled. The simulations are performed with the finite-element solver MSC.Nastran ${ }^{\mathrm{TM}}$ v. 2013, which is able to account for frequency-dependent definitions of material properties. The idea is to distinguish several cases where the viscoelastic properties of the potting resin are changed, while the other parts of the stator's finite-element model are kept identical. In this section, the choice is made to tune only the master-frequency $f_{\eta_{-} \text {max }}$, while the other Zener parameters are fixed with the values measured on resin P3: $G_{0}=2.2 \cdot 10^{7} \mathrm{~Pa}, G_{\infty}=1.4 \cdot 10^{9} \mathrm{~Pa}$ and $\eta_{\max }=0.8$. Although shear moduli are preferred for convenience with the solver's interpretation of viscoelastic properties, the equivalence with Young's moduli is strictly respected through Equation 5, with $v=0.38$. The different cases are labelled with specific IDs, as defined in Table 1.

\begin{tabular}{c|c} 
ID & $f_{\eta_{-} \max }[\mathrm{Hz}]$ \\
\hline $\mathbf{1}$ & 0 \\
$\mathbf{2}$ & $10^{-4}$ \\
$\mathbf{3}$ & $10^{-3}$ \\
$\mathbf{4}$ & $10^{-2}$ \\
$\mathbf{5}$ & $10^{-1}$ \\
$\mathbf{6}$ & 1
\end{tabular}

\begin{tabular}{c|c} 
ID & $f_{\eta_{-} \max }[\mathrm{Hz}]$ \\
\hline $\mathbf{7}$ & $10^{1}$ \\
$\mathbf{8}$ & $10^{2}$ \\
$\mathbf{9}$ & $10^{3}$ \\
$\mathbf{1 0}$ & $10^{4}$ \\
$\mathbf{1 1}$ & $10^{5}$ \\
$\mathbf{1 2}$ & $10^{6}$
\end{tabular}

Table 1: Definitions of the response cases

The value $f_{\eta_{-} \max }=0 \mathrm{~Hz}$ associated to case 1 corresponds to the reference case with frequencyindependent resin properties $G=1.4 \cdot 10^{9} \mathrm{~Pa}, v=0.38$ and $\eta=0$. For cases 2 to 12 , the evolutions of the the potting resin's storage shear modulus and loss factor with respect to frequency are illustrated in Figure 16. It can be clearly seen that the cases for which $f_{\eta \_ \text {max }}$ is below $10^{-2} \mathrm{~Hz}$ are modelled by very similar viscoelastic properties. Also, case 5 represents the current behaviour of resin P3 with $f_{\eta_{-} \text {max }}=10^{-1} \mathrm{~Hz}$, as shown in Figure 5.

The proposed cases may not be processed by the same simulation techniques. The fact that all the material properties are constant in case 1 enables to compute the responses with the modal superposition 

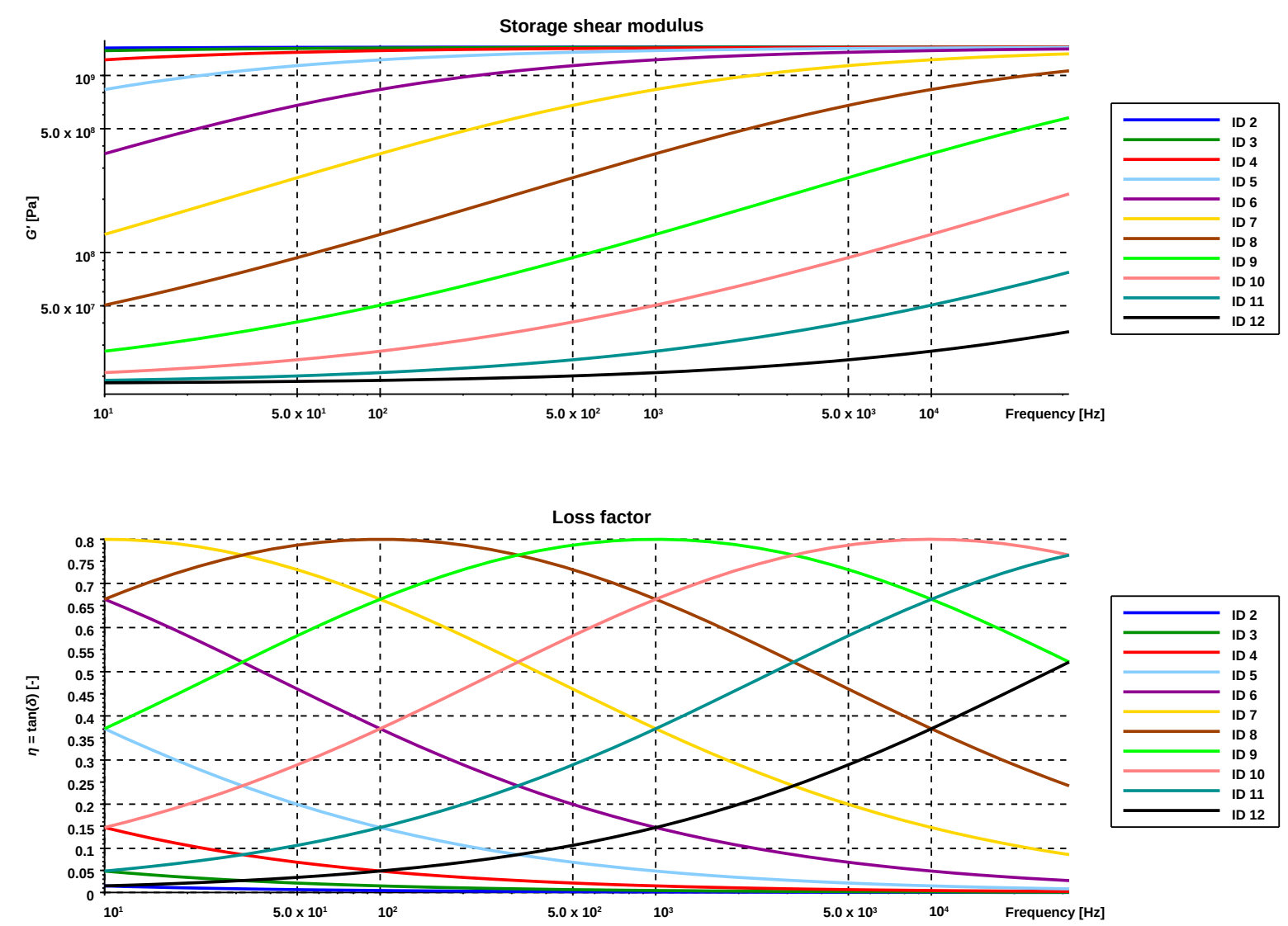

Figure 16: Evolution of the resin's viscoelastic properties (cases 2 to 12)

method [40]. On the contrary, the cases 2 to 12 require direct response solving due to the frequencydependent resin properties, which can be significantly longer than modal superposition (6 minutes for case 1 against 16 hours for each of the cases 2 to 12). This same disadvantage is reported for the iterative complex eigensolution approach developed in [7]. Mitigation solutions exist, such as the pseudo-modal approaches such as in [42] or the numerical reduction methods suggested in [43] or reviewed in [35]. However, each of these alternative choices would require processing the structure's mass and stiffness matrices with external numerical tools, which may be delicate and time-consuming to prepare and perform as the model is composed of about 250,000 degrees of freedom. Therefore, standard direct response solving strategies (as introduced with Equation (1)) are preferred for the cases 2 to 12.

\subsection{Response results of case 1}

Each case introduced in Table 1 is divided into seven sub-cases, as the seven excitation spectra $\mathbf{H}_{8}$ to $\mathbf{H}_{56}$ are applied separately. This is necessary as each excitation order corresponds to a specific frequency range (see Equation 22). The results of case 1 have been transcribed in terms of engine speeds $\mathscr{N}_{r}$ to allow comparison between the different responses. The curves are plotted in Figure 17, where the labels "H $u_{-} \mathrm{r}$ " refer to the respective responses to excitation spectra $\mathbf{H}_{u}$. The dashed curve "RSS_r" is the quadratic sum of the results of all sub-cases $i \in \llbracket 1 ; 7 \rrbracket$, as defined by the following expression: 


$$
R S S\left(\mathscr{N}_{r}\right)=\sqrt{\sum_{i=1}^{7} \operatorname{ERP}\left(\mathscr{N}_{r}\right)^{2}}
$$

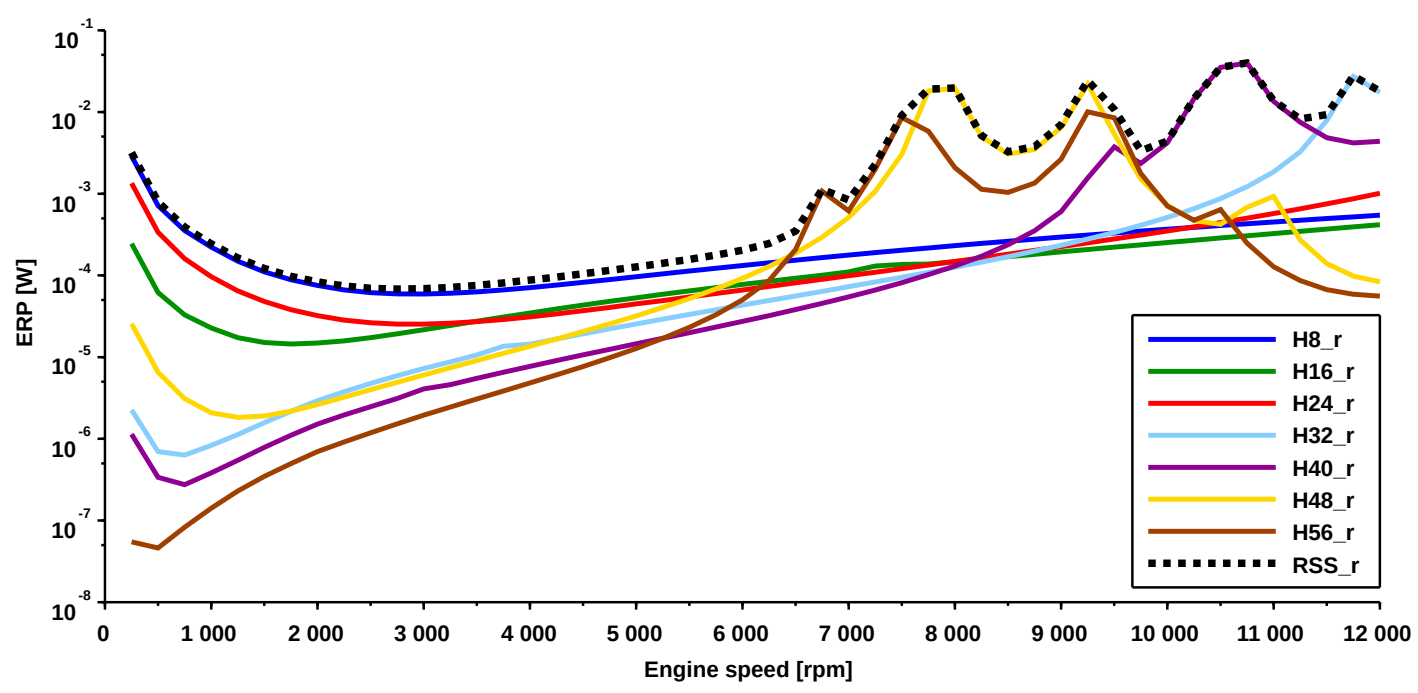

Figure 17: Comparison of ERP responses for case 1

Note that $\operatorname{RSS}\left(\mathscr{N}_{r}\right)$ can be seen basically as an overall sound power level including the contributions $\mathbf{H}_{8}$ to $\mathbf{H}_{56}$. It can be seen that the highest ERP levels are reached in curves H32_r, H40_r, H48_r and H56_r, while lower-order spectra are not responsible of any resonances in this speed range. This can be explained by the fact that for orders $\mathbf{H}_{8}, \mathbf{H}_{16}$ and $\mathbf{H}_{24}$, the engine speeds corresponding to the mode's frequencies are above 12,000 rpm: in spite of the high stress levels illustrated in Figure 12, these orders only create forced responses, whose magnitudes are significantly lower than modal resonances. As for the low engine speeds, the relatively high ERP levels are due to stator's suspension on low-stiffness elements, and should not be regarded as noise problems in real applications.

The peak values on curves H32_r, H40_r, H48_r and H56_r are explained by coincidences with the modes described in Figure 15. The frequencies associated to the peak ERP values are compared to the stator's natural frequencies, whereas the operational deformed shapes are compared to the structure's mode shapes. This comparison is detailed in Table 2.

\begin{tabular}{c||c|c|c|c} 
Response & $\begin{array}{c}\text { Speed } \\
{[\mathrm{rpm}]}\end{array}$ & $\begin{array}{c}\text { Frequency } \\
{[\mathrm{Hz}]}\end{array}$ & $\begin{array}{c}\text { Spatial order of the } \\
\text { excitation contribution }\end{array}$ & $\begin{array}{c}\text { Excited } \\
\text { mode }\end{array}$ \\
\hline H32_r & 11,750 & 6,267 & 0 & $(0,0)$ \\
H40_r & 10,750 & 7,167 & +4 & $(4,0)$ \\
H48_r & 8,000 & 6,400 & 0 & $(0,0)$ \\
& 9,250 & 7400 & 0 & $(0,2)$ \\
H56_r & 7,500 & 7,000 & -4 & $(4,0)$ \\
& 9,250 & 8,633 & -4 & $(4,2)$
\end{tabular}

Table 2: Modal coincidences in case 1

As it has been asserted earlier, Table 2 shows that each of the peak ERP values spotted in the studied 


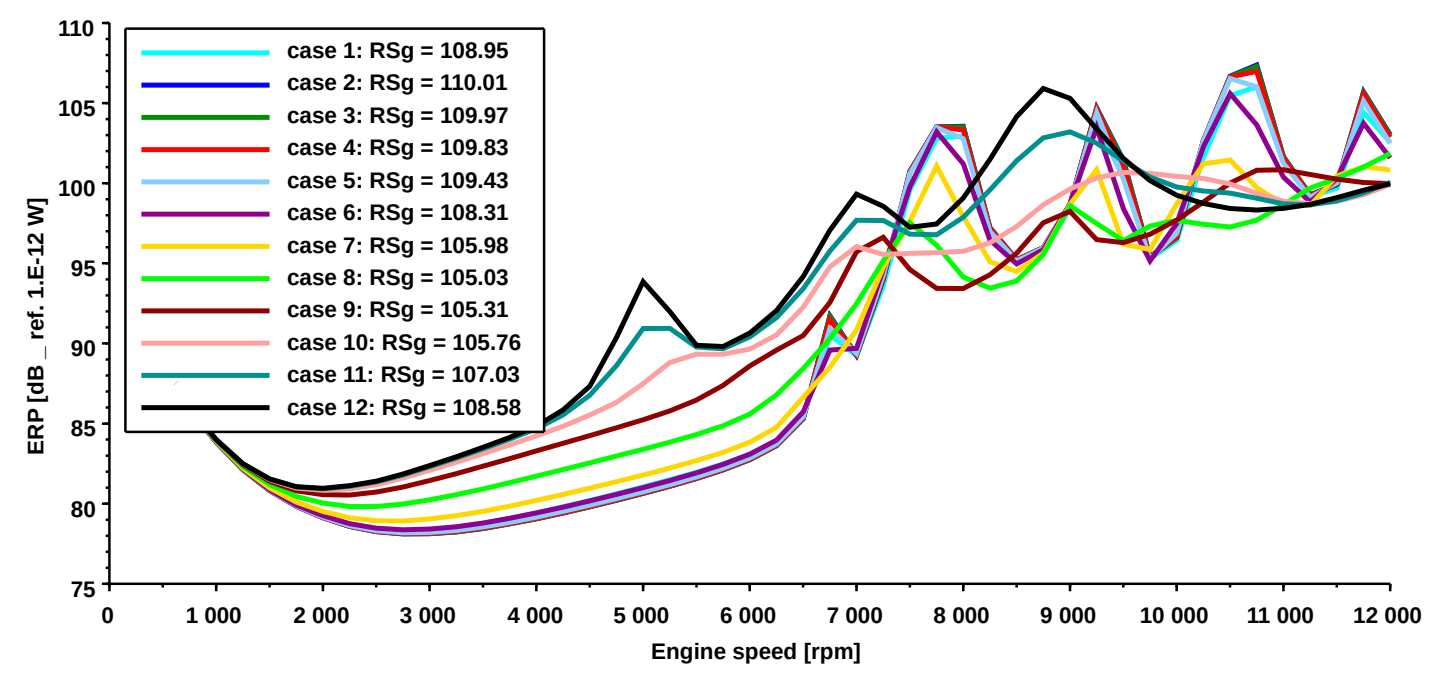

Figure 18: Comparison of the curves $R S S_{\mathrm{dB}}$ for all cases

case coincides in terms of both frequency and deformed shape with one of the structure's critical modes introduced in Figure 15. Except for the sub-case H32_r, these modal coincidences are determined by the excitation spectra's main spatial contributions, which are different according to the load case (see Figure 12).

It can be noted that in H32_r, the resonance at 11,750 rpm coincides with mode $(0,0)$ although the spatial order of the main excitation contribution is -4 ; the frequency of mode $(4,0)$ would have been reached above $13,000 \mathrm{rpm}$ and thus outside the speed range. This resonance is due to the effective excitation of the $(0,0)$ mode by a secondary excitation contribution whose spatial order is 0 .

\subsection{Comparison with other cases}

After every simulation is performed, the ERP responses are processed and a "RSS" curve is computed for each case in order to represent the contributions of all the excitation orders. The values are expressed in $\mathrm{dB}$ according to the expression

$$
\operatorname{RSS}_{\mathrm{dB}}\left(\mathscr{N}_{r}\right)=10 \cdot \log \left(\frac{\operatorname{RSS}\left(\mathscr{N}_{r}\right)}{r e f_{\mathrm{dB}}}\right),
$$

with $r e f_{\mathrm{dB}}=10^{-12} \mathrm{~W}$, and are plotted in Figure 18. In order to compare them, the criterion RSg is computed for each case with

$$
\mathbf{R S g}=10 \cdot \log \left(\frac{\sqrt{\sum R S S\left(\mathscr{N}_{r}\right)^{2}}}{r e f_{\mathrm{dB}}}\right)
$$

Judging from the criterion $\mathbf{R S g}$, the model for which the radiated levels are lowest is case 8, where $f_{\eta_{-} \max }=10^{2} \mathrm{~Hz}$. Compared to case 1 (initial state), the peak response values have been decreased by 5 to $10 \mathrm{~dB}$, which outlines an interesting acoustic opportunity for industrial applications. The results show that ERP maxima are shifted to lower engine speeds as $f_{\eta_{-} \max }$ is shifted to higher frequencies. This is consistent with the shapes of the curves $G^{\prime}(f)$ in Figure 16: increasing $f_{\eta_{-} \text {max }}$ decreases $G^{\prime}$ values and 
thus the structure's natural frequencies. In these conditions, the case $f_{\eta_{-} \max }=10^{2} \mathrm{~Hz}$ can be seen as the best compromise between damping and resonance frequencies for the studied case.

According to the superposition model presented in Subsection 4.2 and described by Equation (17), the glass-transition temperature at $1 \mathrm{~Hz}$ is $T_{G}=79.7^{\circ} \mathrm{C}$, whereas the master-frequency corresponding to the reference temperature $T_{0}=81^{\circ} \mathrm{C}$ is $f_{\eta_{-} \max }=3.29 \mathrm{~Hz}$. Re-arranging Equation (17) such that $f_{\eta_{-} \text {max }}^{\text {nax }}=10^{2} \mathrm{~Hz}$ as found in Section 6 yields:

$$
\log \left(f_{\eta_{-} \max }^{\text {new }}\right)=0.4054 \cdot T_{0}^{\text {new }}-30.834 .
$$

Assuming this new superposition law valid for the optimised resin, the new glass-transition temperature at $1 \mathrm{~Hz}$ will be $T_{G}^{\text {new }}=76.1^{\circ} \mathrm{C}$.

\subsection{Discussion}

So far, this study has optimised the resin's viscoelastic properties by tuning only its representative "master-frequency" $f_{\eta_{-} \max }$. The other Zener parameters could also be thought of for reducing even more the acoustic response of the stator. However, some comments must be outlined in regard of such possible new optimisation steps:

- The resin's mechanical as well as electric and thermal behaviours all depend on the polymer's composition and thus on its components' properties. Due to this dependency towards the monomers' properties, the Zener's asymptotic storage moduli $E_{0}$ and $E_{\infty}$ are also related to the variations of $f_{\eta \_ \text {max }}$. In this study however, as the main excitations (i.e. the radial force resultants) are constant over the considered speed range (see Figure 13), there is no specific need to shift the stator's critical natural frequencies. Therefore, changes of $E_{0}$ and $E_{\infty}$ resulting from changes of $f_{\eta_{-} \max }$ would have negligible impact on the stator's acoustic behaviour as long as no new mode coincidences occur below 11,200 Hz.

- Detecting which excitation orders are likely to lead to modal coincidences prior to the simulation could decrease the computation costs significantly. For example in case 1, the excitation spectra $\mathbf{H}_{8}$, $\mathbf{H}_{16}$ and $\mathbf{H}_{24}$ could have been discarded by predicting that mode coincidences would have occurred beyond 11,200 Hz.

- Other command specifications than imposing a constant global magnetic flux in the motor can be considered. If for instance the strategy had been to impose a constant power in the motor (product of torque and engine speed), the excitation magnitudes would have been inversely proportional to engine speed. In this case, increasing the values of $E_{0}$ and $E_{\infty}$ in the resin would have stiffened the structure and thus shifted the natural frequencies to higher values, where the excitation levels are less significant. This could have helped reducing the overall ERP responses.

- Concerning the maximum damping ratio $\eta_{\max }$, it seems obvious that the higher the value, the smaller magnitude the vibration response would have at the frequency $f_{\eta_{-} \max }$. In terms of Zener models, increasing $\eta_{\text {max }}$ is equivalent to increasing the slope of $E^{\prime}(f)$ in the fast-transition frequency range (see Equations (12) and (13)). Nevertheless, another interesting property to consider is the frequency range over which the resin's master-curves show high damping values (e.g. above $\left.\eta_{\max } / 2\right)$. Designing a direct parameter for tuning this property would thus require another model than Zener's, and could possibly lead to challenging specification for the concrete design of the corresponding polymer. Because of this limitation, optimising the shapes of the curves $\eta(f)$ has not been addressed in this article. 
Eventually, tuning the resin's properties in order to shift the critical modes above $11,200 \mathrm{~Hz}$ could lead to significantly higher asymptotic storage moduli. Although this option is out of the scope of this study, its potential for possible noise reduction will be shortly discussed here.

Let us consider a new polymer $\mathrm{P}_{\text {new }}$ whose storage moduli would be so great that all critical modes would be shifted outside of the study's frequency range. As no mode appropriation would occur anymore, the only vibrational/acoustic phenomena that could be observed would be directly related to the excitations (enforced vibrations).

Comparing this case to the current study could be made by recalculating Equation 27 in the half range $[0 ; 6,000 \mathrm{rpm}]$ (i.e. before the first mode appropriation) and with each $R S S$ term doubled. Taking as example case 2 , for which $R S g\left(\mathrm{P}_{3}\right)=110.1$ (see Figure 18), the new value would be $R S g\left(\mathrm{P}_{\text {new }}\right)=96.5$. This change would not only represent a $12 \%$-drop compared to the resin $\mathrm{P} 3$, but also confine the radiated power to a maximum level (approx. $85 \mathrm{~dB}$ ) which is $20 \mathrm{~dB}$ lower than the resonances observed on modal appropriations.

Although the example presented here seems promising in terms of simulation results, other requirements the resin should fulfill when integrated in an industrial electric machine need to be accounted for (as discussed earlier in this section). Moreover, the storage moduli $E_{0}$ and $E_{\infty}$ the resin should have in order to shift all the critical modes to sufficiently high frequencies may be significantly larger than the usual polymers currently available in the market. Designing and producing such materials might mean increased costs of both development and use that would possibly make them unattractive. These aspects will be analysed in further studies.

\section{Conclusion}

In this article, the optimisation opportunities of a polymer's viscoelastic properties have been investigated for an objective of vibrational and acoustic response reduction in a switched-reluctance machine stator. Focusing on the resin that encapsulates the windings in the stator, master-cruves were computed from experimental dynamic analyses and modelled by analytical Zener models. A temperaturesuperposition analysis enabled to predict the dependence of the polymer in wide ranges of temperature and frequencies. The practical application of this work consisted in simulating realistic electromagnetic excitations and projecting the resulting Maxwell stresses onto the stator's structural finite-element model. Then, the acoustic response could be minimised by tuning the resin's viscoelastic properties, leading to a reduction of peak equivalent radiated power values of 5 to $10 \mathrm{~dB}$.

Within the speed range defined for this application, four "critical" mode coincidences have been outlined as responsible of the peak acoustic responses of the stator. The optimisation of the resin's viscoelastic properties can be therefore seen as the best compromise between mechanical damping and natural frequency shifting for the studied structure.

It seems clear that the results presented in this article are specific to this work; analysing other practical settings with a different polymer to optimise may require developing a similar application. However, the method this article has presented can be applied for any types of structural applications as long as the finite-element simulations take into account the frequency- or temperature-dependent behaviours of the involved viscoelastic materials. The fact that only common finite-element solvers have been used makes this approach easily applicable for other types of concrete industrial applications. As for the design of high-performance resins, attempting to maximise loss tangent values may be a realistic guarantee of vibration level minimisation for many cases.

Eventually, this work presents a new perspective for powering electric vehicles with switched-reluctance machines. Indeed, whereas encapsulating the windings could represent costly operations in the manufac- 
turing process, the acoustic opportunity this method introduces could be decisive in comparison to impregnated stators, where the resin is in lower quantity and has thus a reduced mechanical influence. In addition to this, more advanced polymer designs could be thought of for acoustic level reduction possibilities. For example, polymers with several glass transitions (such as detailed in [44]) could be tuned so that each damping maximum would be placed at frequencies neighbouring the resonances. Future works will focus on analysing the influence of faults on switched-reluctance machines in a similar viscoelastic tuning process, and apply this methodology in other types of electric machines.

\section{Acknowledgements}

The authors would like to thank Mr Nodin and Von Roll for the fruitfull discussions and the practical support provided in this work.

\section{References}

[1] P. Pillay, W. Cai, An investigation into vibration in switched reluctance motors, IEEE Transactions on Industry Applications 35 (3) (1999) 589-596.

[2] W. Tong, Mechanical design of electric motors, Taylor and Francis, 2014.

[3] electrical-engineering portal.com, Side view of an ac motor stator (http://electrical-engineeringportal.com/keeping-motor-windings-dry), accessed on 2015, April $1^{\text {st }}$.

[4] electrical knowhow.com, Winding process of a srm stator (http://www.electricalknowhow.com/2012/05/classification-of-electric-motors-part_22.html), accessed on 2015, April $1^{\text {st }}$.

[5] A.-S. Plouin, É. Balmès, Steel/viscoelastic/steel sandwich shells computational methods and experimental validations, in: International Modal Analysis Conference, Vol. 4062, 2000, pp. 384-390.

[6] J. Slater, W. Keith Belvin, D. Inman, A survey of modern methods for modeling frequency dependent damping in finite element models, in: Proceedings-SPIE - The International Society for Optical Engineering, SPIE INTERNATIONAL SOCIETY FOR OPTICAL, 1993, pp. 1508-1508.

[7] C. Vasques, R. Moreira, J. Rodrigues, Viscoelastic damping technologies - part i: Modeling and finite element implementation, Mechanical Engineering 1 (2) (2010) 96-110.

[8] M. Friswell, D. Inman, Finite element models with viscoelastic damping, in: 17th International Modal Analysis Conference (IMAC), Kissimmee, 1999, pp. 181-187.

[9] A. Tan-Kim, V. Lanfranchi, J. Legranger, F. Palleschi, M. Redon, Influence of temperature on the vibro-acoustic behavior of claw-pole alternators, in: International Conference on Electrical Machines, IEEE, 2014.

[10] W.-Y. Jung, A. Aref, A combined honeycomb and solid viscoelastic material for structural damping applications, Mechanics of materials 35 (8) (2003) 831-844.

[11] A. de Lima, D. Rade, N. Bouhaddi, Optimization of viscoelastic systems combining robust condensation and metamodeling, Journal of the Brazilian Society of Mechanical Sciences and Engineering 32 (SPE) (2010) 485-495. 
[12] M. Trindade, A. Benjeddou, R. Ohayon, Modeling of frequency-dependent viscoelastic materials for active-passive vibration damping, Journal of Vibration and Acoustics 122 (2) (2000) 169-174.

[13] P. Butaud, E. Foltête, M. Ouisse, Sandwich structures with tunable damping properties: On the use of shape memory polymer as viscoelastic core, Composite Structures 153 (2016) 401-408. doi: $10.1016 /$ j.compstruct . 2016.06.040.

[14] H. Endoh, Y. Zama, N. Kadowaki, T. Yatuka, H. Nagai, Y. Mizumura, Viscoelastic resin for vibration damping material, uS Patent 4,859,523 (Aug. 1989).

[15] M. Nakamura, H. Hirakouchi, T. Yatsuka, N. Kadowaki, H. Endoh, Viscoelastic resin composition for vibration-damping material, uS Patent 5,183,863 (Feb. 2 1993).

[16] H. Hirakouchi, M. Nakamura, T. Yatsuka, N. Kadowaki, Viscoelastic resin composition for a vibration damping material, uS Patent 5,411,810 (May 1995).

[17] P. Millithaler, É. Sadoulet-Reboul, M. Ouisse, J.-B. Dupont, N. Bouhaddi, Structural dynamics of electric machine stators: Modelling guidelines and identification of three-dimensional equivalent material properties for multi-layered orthotropic laminates, Journal of Sound and Vibration 348 (2015) 185-205. doi:10.1016/j.jsv.2015.03.010.

[18] P. Millithaler, Dynamic behaviour of electric machine stators: modelling guidelines for efficient finite-element simulations and design specifications for noise reduction, Ph.D. thesis, University of Franche-Comté, Besançon (2015).

[19] P. Millithaler, E. Sadoulet-Reboul, M. Ouisse, J.-B. Dupont, N. Bouhaddi, Identification of representative anisotropic material properties accounting for friction and preloading effects: A contribution for the modeling of structural dynamics of electric motor stators, Journal of Vibration and Controldoi: 10.1177/1077546316637941.

[20] C. Schlensok, B. Schmülling, M. Van der Giet, K. Hameyer, Electromagnetically excited audible noise-evaluation and optimization of electrical machines by numerical simulation, Journal of Electrical Power Quality and Utilisation 26 (3) (2006) 727-742.

[21] L. Humbert, P. Pellerey, S. Cristaudo, Electromagnetic and structural coupled simulation to investigate nvh behavior of an electrical automotive powertrain, SAE International (12SNVH-0002) (2012) $1-12$.

[22] J.-B. Dupont, P. Bouvet, J.-L. Wojtowicki, Simulation of the airborne and structure-borne noise of electric powertrain, validation of the simulation methodology, SAE International.

[23] S. Kaliappan, Characterization of physical properties of polymers using afm force-distance curves, Ph.D. thesis, Chemie-Biologie Fachbereich der Universität Siegen (2007).

[24] J. Ferry, Viscoelastic Properties of Polymers, 3rd edition, 1980.

[25] L. Rouleau, Modélisation vibro-acoustique de structures sandwich munies de matériaux viscoélastiques., Ph.D. thesis, Conservatoire National des Arts et Métiers (2013).

[26] J. Nicholson, The chemistry of polymers, Royal Society of Chemistry, 2011. 
[27] K. Bouayed, P. Mordillat, L. Mebarek, M. Hamdi, Numerical modelling of the vibro-acoustic behavior of a closed vehicle with frequency dependent polymer materials, in: INTER-NOISE and NOISE-CON Congress and Conference Proceedings, Vol. 249, Institute of Noise Control Engineering, 2014, pp. 5685-5693.

[28] A. Galucio, J.-F. Deü, R. Ohayon, Finite element formulation of viscoelastic sandwich beams using fractional derivative operators, Computational Mechanics 33 (4) (2004) 282-291.

[29] P. Butaud, M. Ouisse, V. Placet, E. Foltête, Experimental investigations on viscoelastic properties of a shape memory polymer, in: ASME 2014, Conference on Smart Materials, Adaptive Structures and Intelligent Systems, American Society of Mechanical Engineers, 2014.

[30] E. Hearn, Mechanics of Materials, Vol. 1 - 2, Pergamon Press, 1985.

[31] J. Rieger, The glass transition temperature tg of polymers - comparison of the values from differential thermal analysis (dta, dsc) and dynamic mechanical measurements (torsion pendulum), Polymer testing 20 (2) (2001) 199-204.

[32] J. Capodagli, R. Lakes, Isothermal viscoelastic properties of pmma and ldpe over 11 decades of frequency and time: a test of time-temperature superposition, Rheologica Acta 47 (7) (2008) 777786.

[33] E. Chailleux, G. Ramond, C. Such, C. de La Roche, A mathematical-based master-curve construction method applied to complex modulus of bituminous materials, Road Materials and Pavement Design 7 (sup1) (2006) 75-92.

[34] N. Yusoff, E. Chailleux, G. Airey, A comparative study of the influence of shift factor equations on master curve construction, Tech. Rep. 6, Chinese Society of Pavement Engineering (2011).

[35] L. Rouleau, J.-F. Deü, A. Legay, J.-F. Sigrist, Vibro-acoustic study of a viscoelastic sandwich ring immersed in water, Journal of Sound and Vibration 331 (3) (2012) 522-539.

[36] J. Dealy, D. Plazek, Time-temperature superposition - a users guide, Rheology Bulletin 78 (2) (2009) $16-31$.

[37] D. Dorrell, M.-F. Hsieh, Calculation of radial forces in cage induction motors at start - the effect of rotor differential, IEEE Transactions on Magnetics 46 (8) (2010) 3029-3032.

[38] P. Pellerey, V. Lanfranchi, G. Friedrich, Coupled numerical simulation between electromagnetic and structural models. influence of the supply harmonics for synchronous machine vibrations, IEEE Transactions on Magnetics 48 (2) (2012) 983-986.

[39] K. Delaere, R. Belmans, K. Hameyer, W. Heylen, P. Sas, Coupling of magnetic analysis and vibrational modal analysis using local forces, in: $\mathrm{X}^{\text {th }}$ International Symposium on Theoretical Electrical Engineering ISTET, Vol. 99, 1999, pp. 417-422.

[40] R. Craig, Structural dynamics - an introduction to computer methods, Vol. 40, Wiley New York, 1981.

[41] MSC Software, MSC Nastran Dynamic Analysis User's Guide, p. 752 (2012). 
[42] A.-S. Plouin, É. Balmès, Pseudo-modal representations of large models with viscoelastic behavior, in: Society for Experimental Mechanics, Inc, 16th International Modal Analysis Conference, Vol. 2, 1998, pp. 1440-1446.

[43] M. Friswell, D. Inman, Reduced-order models of structures with viscoelastic components, AIAA journal 37 (10) (1999) 1318-1325.

[44] J. Fernández-Blázquez, A. Bello, E. Pérez, Dynamic mechanical analysis of the two glass transitions in a thermotropic polymer, Polymer 46 (23) (2005) 10004-10010. 